ARTICLE

Received 23 Aug 2015 | Accepted 13 Oct 2016 | Published 23 Nov 2016

DOI: $10.1038 /$ ncomms13546 OPEN

\title{
PPFIA1 drives active $\alpha 5 \beta 1$ integrin recycling and controls fibronectin fibrillogenesis and vascular morphogenesis
}

Giulia Mana ${ }^{1,2, \star}$, Fabiana Clapero ${ }^{1,2, \star}$, Emiliano Panieri ${ }^{3}$, Valentina Panero ${ }^{3}$, Ralph T. Böttcher ${ }^{4}$, Hui-Yuan Tseng ${ }^{4}$, Federico Saltarin ${ }^{1,2}$, Elena Astanina ${ }^{1,5}$, Katarzyna I. Wolanska ${ }^{6}$, Mark R. Morgan ${ }^{6}$, Martin J. Humphries ${ }^{7}$, Massimo M. Santoro ${ }^{3,8}$, Guido Serini ${ }^{1,2,{ }^{* \star}} \&$ Donatella Valdembri ${ }^{1,2,{ }^{* \star}}$

Basolateral polymerization of cellular fibronectin (FN) into a meshwork drives endothelial cell (EC) polarity and vascular remodelling. However, mechanisms coordinating $\alpha 5 \beta 1$ integrin-mediated extracellular FN endocytosis and exocytosis of newly synthesized FN remain elusive. Here we show that, on Rab21-elicited internalization, $\mathrm{FN}$-bound/active $\alpha 5 \beta 1$ is recycled to the EC surface. We identify a pathway, comprising the regulators of post-Golgi carrier formation PI4KB and AP-1A, the small GTPase Rab11B, the surface tyrosine phosphatase receptor PTPRF and its adaptor PPFIA1, which we propose acts as a funnel combining $\mathrm{FN}$ secretion and recycling of active $\alpha 5 \beta 1$ integrin from the trans-Golgi network (TGN) to the EC surface, thus allowing FN fibrillogenesis. In this framework, PPFIA1 interacts with active $\alpha 5 \beta 1$ integrin and localizes close to EC adhesions where post-Golgi carriers are targeted. We show that PPFIA1 is required for FN polymerization-dependent vascular morphogenesis, both in vitro and in the developing zebrafish embryo.

\footnotetext{
${ }^{1}$ Department of Oncology, University of Torino School of Medicine, Candiolo, Torino 10060, Italy. ${ }^{2}$ Laboratory of Cell Adhesion Dynamics, Candiolo Cancer Institute-Fondazione del Piemonte per I'Oncologia (FPO), Istituto di Ricovero e Cura a Carattere Scientifico (IRCCS), Candiolo, Torino 10060, Italy. ${ }^{3}$ Department of Molecular Biotechnology and Health Sciences, Molecular Biotechnology Center, University of Torino, 10126 Torino, Italy. ${ }^{4}$ Department of Molecular Medicine, Max Planck Institute of Biochemistry, Martinsried 82152, Germany. ${ }^{5}$ Laboratory of Vascular Oncology, Candiolo Cancer Institute-Fondazione del Piemonte per l'Oncologia (FPO), Istituto di Ricovero e Cura a Carattere Scientifico (IRCCS), Candiolo, Torino 10060, Italy. ${ }^{6}$ Department of Cellular and Molecular Physiology, Institute of Translational Medicine, University of Liverpool, Liverpool L69 3BX, UK. ${ }^{7}$ Wellcome Trust Centre for Cell-Matrix Research, Faculty of Biology, Medicine \& Health, University of Manchester, Manchester M13 9PT, UK. ${ }^{8}$ Laboratory of Endothelial Molecular Biology, Vesalius Research Center, VIB, Leuven B-3000, Belgium. ${ }^{\star}$ These authors contributed equally to this work. ${ }^{\star \star}$ These authors jointly supervised this work. Correspondence and requests for materials should be addressed to G.S. (email: guido.serini@ircc.it) or to D.V. (email: donatella.valdembri@ircc.it).
} 
E ssentially all morphogenetic events of multicellular organisms, including angiogenesis ${ }^{1}$, require a highly dynamic adhesion to extracellular matrix (ECM) proteins that is mediated primarily by integrins ${ }^{2}$. During vascular development, the interaction between fibronectin (FN) and its major receptor $\alpha 5 \beta 1$ integrin induces apico-basal polarity of endothelial cells (ECs) and the formation of the single lumen of blood vessels ${ }^{3,4}$. Integrin-mediated ECM signalling controls the spatial orientation of the apico-basal axis not only in $\mathrm{ECs}^{5}$, but also in several other polarized cell types ${ }^{6}$. Cells secrete FN as a soluble dimer that is assembled by integrins into a fibrillar network. The latter represents the bioactive form of FN and is capable of inducing mechanical and chemical signals that are essential for EC tubulogenesis ${ }^{7}$ and in vivo angiogenesis ${ }^{1,8}$. Although the fibrillar FN network is cross-linked and associated with other ECM proteins ${ }^{8}$, it is continuously turned over and remodelled due to matrix metalloproteinase-dependent cleavage ${ }^{9}$ and integrin-mediated endocytosis of freed FN proteolytic fragments ${ }^{10-13}$. Indeed, a complex machinery composed of the transmembrane glycoprotein neuropilin 1 (Nrp1), its cytosolic interactor GAIP interacting protein $\mathrm{C}$ terminus, member 1 (GIPC1) and the associated motor myosin VI (MYO6) selectively controls the endocytosis of FN fragments bound to active $\alpha 5 \beta 1$ integrins from the surface of ECs ${ }^{11,14}$. The importance of this process is highlighted by the severe impairment of FN fibrillogenesis that occurs in ECs on disruption of Nrp1 signaling ${ }^{14}$. Since internalized active $\alpha 5 \beta 1$ integrins recycle back to the cell surface ${ }^{15,16}$, it has been proposed that they may also accomplish other tasks in addition to the endocytosis of FN fragments, such as the binding of newly synthesized FN in the exocytosis pathway, resulting in its deposition and polymerization at the basolateral surface of ECs to replenish degraded FN fibrils ${ }^{9,10,14}$. Despite the attractiveness of this hypothesis and the fact that the turnover of the fibrillar FN network is essential for physiologic as well as pathologic angiogenesis, it is unknown where in the cell $\alpha 5 \beta 1$ integrins may be loaded with newly synthesized FN molecules and how the exocytosis of the FN- $\alpha 5 \beta 1$ integrin complex could be orchestrated at the molecular level.

In polarized cells, the asymmetric distribution of transmembrane and secreted soluble proteins, for example, the selective basolateral deposition of FN fibrils in ECs ${ }^{17-19}$, relies on the unique sorting properties of the TGN along the biosynthetic routes $^{20,21}$. Specific apical and basal endosomal compartments are thought to control the local recycling of endocytosed receptors ${ }^{22}$. However, recent studies suggest that internalized integrins may also reach the TGN before being recycled back to the plasma membrane ${ }^{23-26}$. Liprins $\alpha$ and $\beta$ (ref. 27) are widely expressed and related adaptor proteins that, by interacting with the protein tyrosine phosphatase receptor type $\mathrm{f}$ polypeptide (PTPRF, also known as leukocyte common antigen related-LAR protein $)^{28}$, were originally found to control synapse formation and function ${ }^{29,30}$. Notably, in addition to cooperating with liprin $\beta$ in the control of synapse size ${ }^{30}$, PTPRF interacting protein $\alpha 1$ (PPFIA1), otherwise known as liprin- $\alpha 1$ (ref. 27), also coordinates presynaptic endo-exocytic traffic and drives the docking of neurotransmitter-containing vesicles with the plasma membrane ${ }^{29,31,32}$. Here, we identify PPFIA1 as a key component of the molecular machinery that in ECs physically binds and controls the recycling of endocytosed active $\alpha 5 \beta 1$ integrin to the cell surface and the basolaterally-polarized fibrillogenesis of newly synthesized FN.

\section{Results}

PPFIA1 binds to and controls active $\alpha 5 \beta 1$ integrin recycling. We have previously identified Nrp1 and additional adaptor and motor proteins as key components of a signalling complex that selectively promotes endocytosis of active $\alpha 5 \beta 1$ integrin and the polymerization of FN into a basolateral fibrillar network by $\mathrm{ECs}^{11,14}$. While these findings highlight endocytic trafficking events involving active $\alpha 5 \beta 1$ integrins ${ }^{11,14}$ for the turnover of FN fibrils ${ }^{9,10}$, it is unclear whether the endocytosed active $\alpha 5 \beta 1$ integrins only remove fibrillar FN or whether they also replenish it. To address this question, we determined the subcellular localization of the ubiquitously expressed adaptor protein PPFIA1 that has been shown to couple endocytosis and exocytosis in presynaptic nerve termini ${ }^{32}$. Standard confocal microscopy analysis revealed that, as previously reported ${ }^{33}$, fluorescently immunostained PPFIA1 localized around mature peripheral focal adhesions of ECs (Fig. 1a, magnified panel 1). Furthermore, PPFIA1 also localized to the vicinity of centrally located vinculincontaining fibrillar adhesions (Fig. 1a, magnified panel 2), where active $\alpha 5 \beta 1$ integrins, as recognized by SNAKA51 monoclonal antibody $(\mathrm{mAb})^{34,35}$, predominantly localized ${ }^{14}$. Similarly, when transfected in ECs, PPFIA1-GFP concentrated in close proximity to focal (Fig. 1b, magnified panel 1) and fibrillar (Fig. 1b, magnified panel 2) adhesions. These findings were further substantiated by super-resolved timed-gated stimulated emission depletion (g-STED) confocal microscopy analyses of PPFIA1-GFP transfected and anti-vinculin-stained or anti-active $\alpha 5 \beta 1$ integrin-stained ECs (Fig. 1a,b, g-STED).

To test whether PPFIA1 represents a novel regulator of active $\alpha 5 \beta 1$ integrin recycling, we first assessed whether PPFIA1 binds $\alpha 5 \beta 1$ integrins, and if NRP1 mediates this interaction. Lysates from ECs oligofected with either control siRNA (siCTL) or a pool of four siRNA targeting NRP1 (siNRP1) were immunoprecipitated with mAbs recognizing either total (that is, active and inactive, clone VC5) or active (clone SNAKA51) $\alpha 5 \beta 1$ integrins $^{36}$ (Fig. 1c, left panel). PPFIA1 co-immunoprecipitated with SNAKA $51^{+}$/active $\alpha 5 \beta 1$ integrins, but NRP1 did not mediate the interaction (Fig. 1d). Indeed, quantitative confocal analysis of relative surface fluorescence of individual ECs showed that SNAKA $51^{+}$active $\alpha 5$ integrin subunits account for the $10 \%$ of $\mathrm{VC}^{+}$or $\mathrm{AB} 1928^{+}$total $\alpha 5$ integrin subunits (Supplementary Fig. 1).

To corroborate the interaction between active $\alpha 5 \beta 1$ integrin and PPFIA1, we performed pull-down assays from cell lysates using recombinantly expressed and purified monomeric or dimeric $\alpha 5$ or $\beta 1$ integrin cytotails. Complementary DNAs encoding the cytoplasmic domain of either $\alpha 5$ or $\beta 1$ integrin subunit were fused at their N-terminus with Jun and Fos peptides, respectively (Fig. 1c, right panel). To mimic the unclasped and active or the clasped and inactive $\alpha 5 \beta 1$ integrin conformation, recombinant $\alpha 5$ and $\beta 1$ cytotails were employed alone or in combination to force their dimerization and clasping via the high affinity Jun-Fos interaction (Fig. 1c, right panel). The well-known $\beta 1$ integrin cytosolic interactor kindlin 2 (ref. 37) was used as a positive control. Both PPFIA1 and kindlin 2 were pulled down by isolated Fos $\beta 1$, but not Jun $\alpha 5$ integrin subunit cytotails. Furthermore, the interactions of both proteins were significantly impaired by forced Jun-Fos-mediated dimerization of $\alpha 5$ and $\beta 1$ integrin subunit cytotails (Fig. 1e).

In ECs, PPFIA1 localizes in close proximity to fibrillar adhesions and interacts with the $\beta 1$ cytotail of active $\alpha 5 \beta 1$ integrins. Hence, we sought to investigate whether, in line with the role it plays in neuronal synapses ${ }^{31,32}$, PPFIA1 participates in the recycling of endocytosed active $\alpha 5 \beta 1$ integrins. By means of biochemical assays ${ }^{38}$, we first analyzed the extent of total or active $\alpha 5 \beta 1$ integrin recycling at different time points on internalization. In accordance with previous quantitative fluorescence confocal microscopy analyse ${ }^{39}$, we found that total $\alpha 5 \beta 1$ integrin rapidly recycled at early $(5 \mathrm{~min})$, but not late $(10-15 \mathrm{~min})$ time 
points after internalization (Supplementary Fig. 2a). By contrast, active $\alpha 5 \beta 1$ integrin was more efficiently recycled back to the EC surface at late $(15 \mathrm{~min})$ rather than early $(5 \mathrm{~min})$ time points after internalization (Supplementary Fig. 2b). Next, we studied the effects of PPFIA1 silencing (siPPFIA1, Fig. 2a) on active $\alpha 5 \beta 1$ integrin recycling in ECs. pLVX lentivirus-driven overexpression of an siRNA-resistant PPFIA1 (pLVX-PPFIA1r) was employed to rescue PPFIA1 expression levels (Fig. 2a). Notably, when
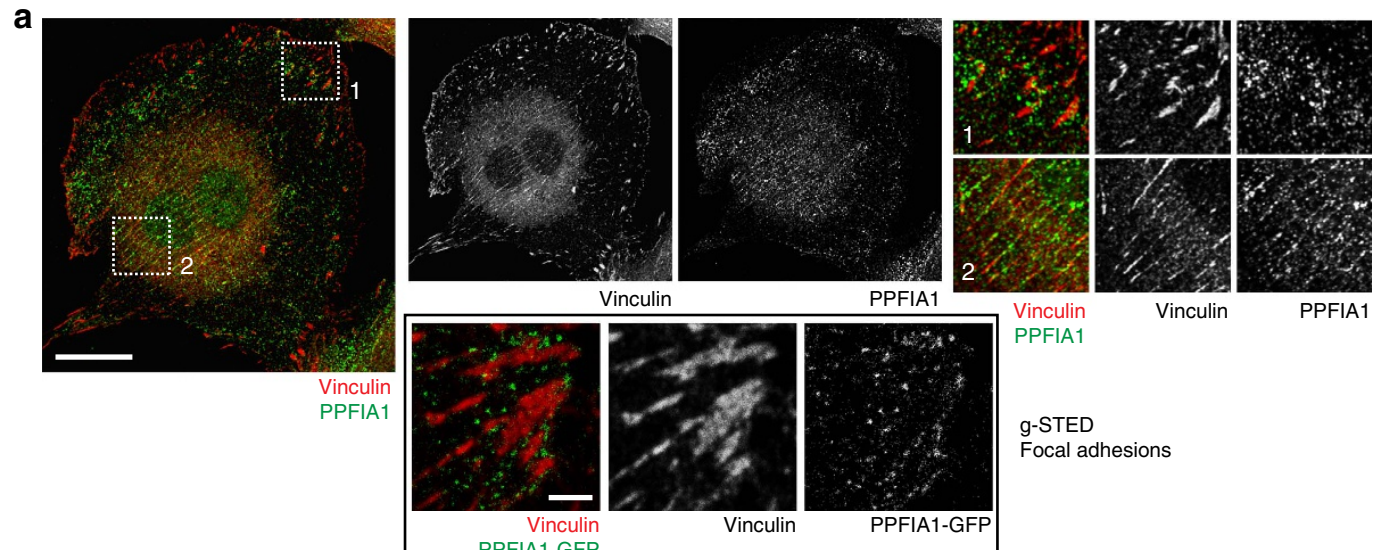

PPFIA1

PPFIA1 PPFIA1-GFP
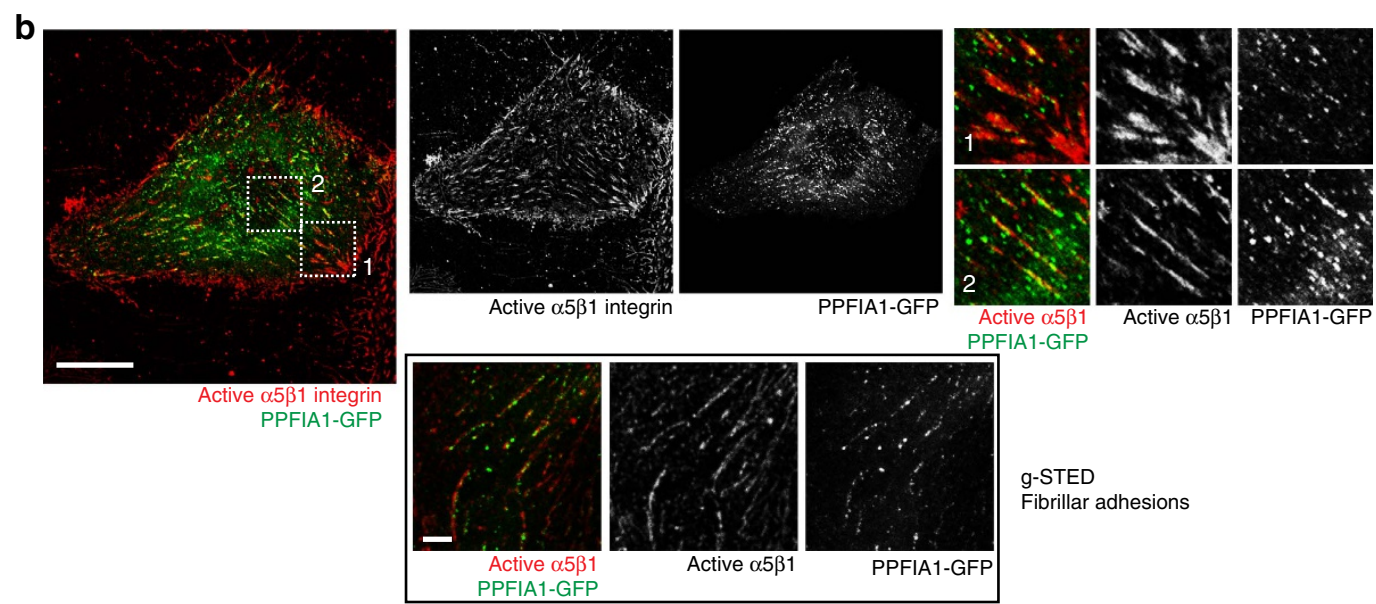

g-STED

Focal adhesions PPFIA1-GFP
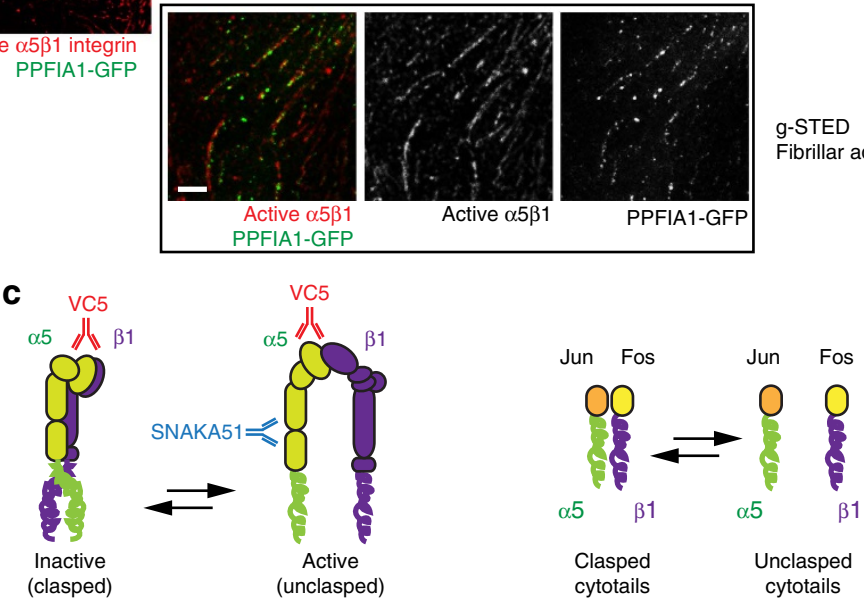

d

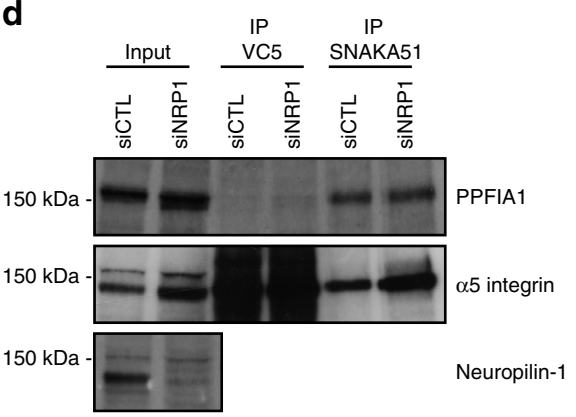

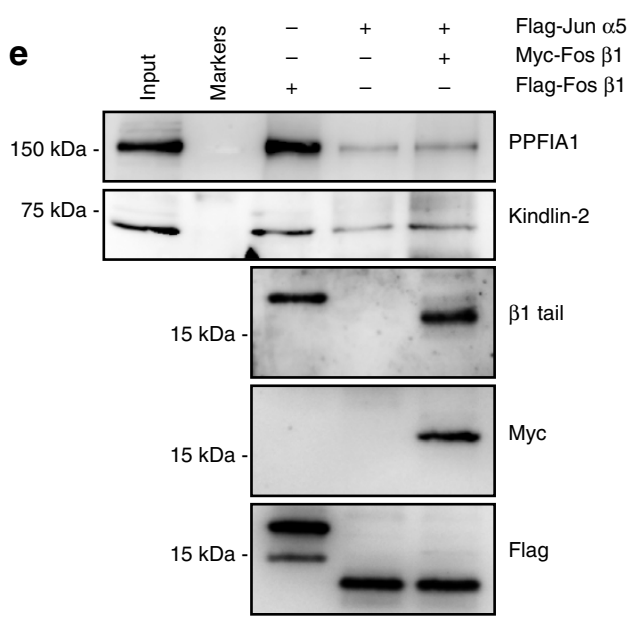


compared with control transduced (pLVX) and silenced (siCTL) ECs, PPFIA1 knockdown (pLVX siPPFIA1) significantly reduced the amount of recycled active $\alpha 5 \beta 1$ integrin (Fig. $2 \mathrm{~b}$ ). Importantly, pLVX-PPFIA1r restored the defective recycling phenotype of PPFIA1-silenced (pLVX-PPFIA1r siPPFIA1) ECs (Fig. 2b).

To determine whether impaired active $\alpha 5 \beta 1$ integrin recycling may correlate with its altered subcellular localization, we incubated SNAKA51 mAb for $20 \mathrm{~min}$ at $37^{\circ} \mathrm{C}$ on confluent living PLVX siCTL or PLVX siPPFIA1 ECs that were then processed for immunofluorescence and analyzed by confocal microscopy. In comparison with pLVX siCTL ECs, where SNAKA51 mainly localized in $\alpha 5 \beta 1$-containing fibrillar adhesions, in pLVX siPPFIA1 ECs SNAKA51 ${ }^{+} /$active $\alpha 5 \beta 1$ integrins accumulated in perinuclear punctae (Fig. 2c). Reintroduction of PPFIA1r rescued the fibrillar distribution of active $\alpha 5 \beta 1$ integrin in PPFIA1-silenced (pLVX-PPFIA1r siPPFIA1) ECs (Fig. 2c). Furthermore, fluorescence confocal microscopy analyses of perinuclear punctae revealed that, in siPPFIA1 ECs, internalized active $\alpha 5 \beta 1$ integrin accumulated in $\mathrm{TGN}_{4}{ }^{+}$vesicles, which correspond to bona fide post-Golgi carriers (PGCs) and, in accordance with previous findings ${ }^{40,41}$, are often positive for the early endosome antigen 1 (EEA1) (Fig. 2d, upper panels). The negligible fraction of internalized SNAKA51 $1^{+}$active $\alpha 5 \beta 1$ integrins that colocalize with the late endosome marker lysosomal-associated membrane protein 1 (LAMP1) was not affected by PPFIA1 silencing (Fig. 2d, lower panels).

Unlike non-polarized fibroblasts ${ }^{42}$, FN fibrils are deposited beneath the basolateral surface of polarized ECs ${ }^{17-19}$. Similarly to what has been previously reported for active $\alpha \mathrm{v} \beta 3$ integrin after shear stress ${ }^{43}$, quantitative analysis of apico-basal mean intensity ratio by confocal $\mathrm{xz}$ sectioning on both sparse (Supplementary Fig. 3) and confluent ECs (Fig. 3a-c) revealed that SNAKA51+/active $\alpha 5 \beta 1$ integrins localize along the basolateral side of pLVX siCTL ECs, while in pLVX siPPFIA1 ECs they were randomly redistributed around the cell surface (Fig. 3a-c). Also in this case, lentiviral transduction of PPFIA1r rescued the defective polarized membrane distribution of SNAKA $51^{+}$/active $\alpha 5 \beta 1$ integrin caused by PPFIA1 silencing in ECs (Fig. 3a-c). Furthermore, confocal $x z$ sectioning demonstrated that also PPFIA1-GFP resides along the basolateral side of confluent ECs (Supplementary Fig. 4).

Taken together, these data indicate that in ECs PPFIA1 selectively associates with and regulates the trafficking of SNAKA $51^{+}$/active $\alpha 5 \beta 1$ integrins.

PPFIA1 controls FN secretion and polymerization. Since $\alpha 5 \beta 1$ integrin is the principal FN receptor in ECs and its localization in fibrillar adhesions is fundamental to funnel actomyosin tension necessary to unfold and incorporate secreted FN dimers into polymeric fibrils ${ }^{8}$, we asked whether the polymerization of extra domain-A (ED-A)-containing cellular FN (ED-A FN) was controlled by PPFIA1. Confluent pLVX siCTL, pLVX siPPFIA1 and pLVX-PPFIA1r siPPFIA1 ECs were cultured in medium containing plasma FN-depleted fetal bovine serum (FBS) and endogenous ED-A FN was visualized with the IST9 $\mathrm{mAb}$ and confocal fluorescence microscopy. While pLVX siCTL ECs polymerized ED-A FN into an extracellular fibrillar network (Fig. 4a, upper panels), which confocal xz sectioning localized to the basolateral membranes (Fig. 4b), pLVX siPPFIA1 ECs accumulated FN in a perinuclear compartment inside ECs (Fig. 4a, middle panels). Importantly, pLVX-mediated PPFIA1r overexpression strongly decreased ED-A FN intracellular accumulation and restored its polymerization (Fig. 4a, lower panels). Addition of exogenous FN to the medium did not rescue the defective fibrillogenesis of endogenous ED-A FN in PPFIA1-silenced ECs (Supplementary Fig. 5).

Next, we investigated whether the subcellular compartment in which ED-A FN accumulated on PPFIA1 silencing overlapped with the compartment(s) in which active $\alpha 5 \beta 1$ integrins accumulated in siPPFIA1 ECs. To this end, living cells were incubated with SNAKA5 $1 \mathrm{mAb}$ at $37^{\circ} \mathrm{C}$ for $20 \mathrm{~min}$, fixed, stained and analyzed by fluorescence confocal microscopy. Interestingly, in contrast to pLVX siCTL ECs, pLVX siPPFIA1 ECs displayed a large accumulation of ED-A FN in TGN46 ${ }^{+}$TGN cisternae. Furthermore, ED-A FN staining overlapped with internalized SNAKA51 mAb-bound, active $\alpha 5 \beta 1$ integrin only in TGN46 ${ }^{+}$ vesicular PGCs, but not in TGN cisternae of pLVX siPPFIA1 ECs (Fig. 4c). Moreover, exogenously added rhodamine-labelled plasma FN reached the $\mathrm{TGN}^{+} 6^{+}$positive PGCs of siPPFIA1 ECs, but did not enter the TGN cisternae where instead endogenous ED-A FN accumulated (Fig. 4d). Next, we explored if PPFIA1 silencing affects not only the assembly of ED-A FN into a basolateral fibrillar meshwork, but also its polarized secretion. To this end, we set up a quantitative biochemical apico-basal secretion assay that, through the use of Transwell polycarbonate membrane inserts, allowed quantification of the amount of soluble ED-A FN that confluent ECs released in either the apical or basolateral culture medium. In keeping with previous findings ${ }^{17-19}$, we observed that the secretion of endogenous ED-A FN mainly occurred at the basolateral side of ECs (Fig. 4e). Notably, PPFIA1 silencing severely impaired basolateral, but not apical, ED-A FN secretion in the medium of confluent cultured ECs (Fig. 4e).

Altogether, these data show how in ECs the lack of PPFIA1 simultaneously impairs the recycling of endocytosed active $\alpha 5 \beta 1$ integrin and the basolateral secretion of endogenous ED-A FN.

Figure 1 | PPFIA1 localizes in proximity of EC fibrillar adhesions and selectively interacts with active $\boldsymbol{\alpha} \mathbf{5 \beta 1}$ integrin cytodomain. (a,b) Conventional or g-STED (boxed) confocal microscopy analyses of endogenous PPFIA1 (green) or PPFIA1-GFP (green) and vinculin (red) or SNAKA51+ active $\alpha 5 \beta 1$ integrin (red) in ECs indicates that PPFIA1 localizes close to both focal and fibrillar adhesions. Right-sided panels 1 (focal adhesions) and $\mathbf{2}$ (fibrillar adhesions) are magnifications of boxed areas in left-sided panels. (c) Representation of inactive and active $\alpha 5 \beta 1$ integrin displaying clasped or unclasped cytotails, respectively. Epitopes recognized by VC5 and SNAKA51 mAbs are shown. VC5 binds to a $\beta$ propeller domain epitope of the $\alpha 5$ subunit of both inactive and active $\alpha 5 \beta 1$ integrin, while SNAKA51 binds to the calf domains of the $\alpha 5$ subunit only when $\alpha 5 \beta 1$ integrin is active. Jun or Fos N-terminally-tagged recombinant integrin cytotails were exploited to mimic $\alpha 5 \beta 1$ integrin activation state. Jun-Fos dimerization and clasping of integrin cytotails, mimicked inactive $\alpha 5 \beta 1$ integrin. (d) Immunoprecipitation from EC lysates of total (that is, inactive and active) or active $\alpha 5 \beta 1$ integrin by VC5 and SNAKA51 mAbs, respectively, followed by western blotting with anti-PPFIA1 or anti- $\alpha 5$ integrin subunit or anti-Nrp1 antibodies. In ECs, PPFIA1 associates with active, but not inactive $\alpha 5 \beta 1$ integrin in both siCTL and siNRP1 ECs. Total EC lysates (input) were employed for control purposes. (e) Pull-down from mouse fibroblast cell lysates of PPFIA1 on isolated or dimerized $\alpha 5$ or $\beta 1$ integrin cytotails. Kindlin- 2 was pulled down for control purposes. Both PPFIA1 and kindlin-2 are pulled down on isolated Myc-Fos $\beta 1$, but not Flag-Jun $\alpha 5$ integrin subunit cytotail. Furthermore, both interactions are severely impaired by forced Jun-Fos-mediated dimerization of $\alpha 5$ and $\beta 1$ integrin subunit cytotails. $\alpha 5$ or $\beta 1$ integrin cytotails are revealed by means of Western Blot with the respective anti-tag antibodies (Flag and/or Myc) or anti- $\beta 1$ integrin subunit cytotail (for loading control purposes). Scale bar, $20 \mu \mathrm{m}(\mathbf{a}, \mathbf{b})$ and $2 \mu \mathrm{m}$ (boxed g-STED panels). 
Furthermore, PPFIA1 silencing results in an abnormal accumulation of both ED-A FN and endocytosed active $\alpha 5 \beta 1$ integrin in PGCs. With respect to the role of PPFIA1 in the docking of secretory vesicles to the plasma membrane of neuronal presynaptic terminals ${ }^{31,32}$, we asked whether TGN46 ${ }^{+}$PGCs might also reach the basolateral plasma membrane of ECs and localize in close proximity to adhesion sites where PPFIA1 concentrates (Fig. 1; ref. 33). Confocal microscopy analysis on fixed ECs revealed a sizeable accumulation of TGN46 ${ }^{+}$PGC vesicles around vinculin-containing adhesion sites where PPFIA1 a

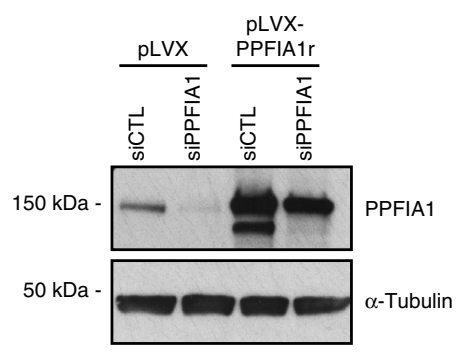

C
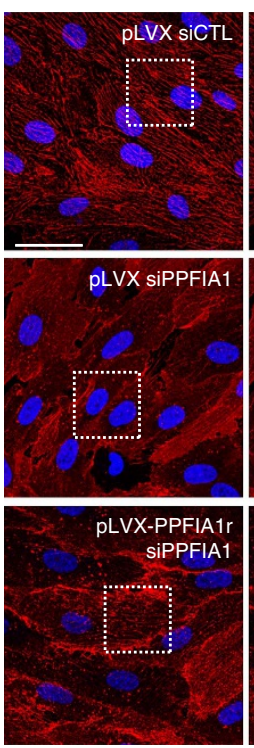

DAPI

Active $\alpha 5 \beta$
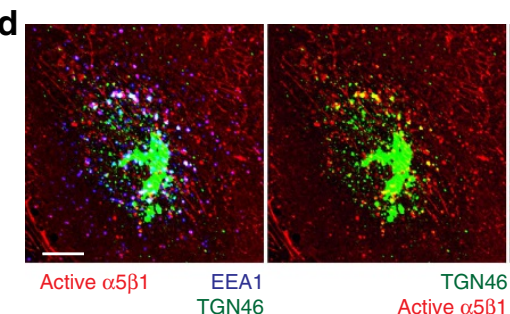

GN46
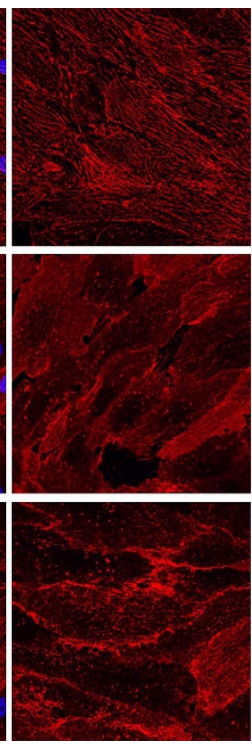

Active $\alpha 5 \beta 1$
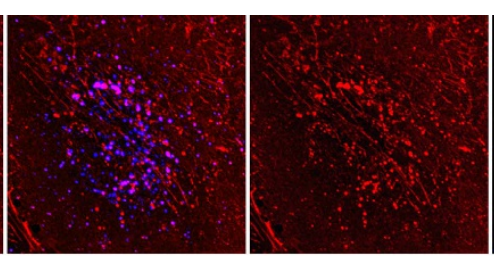

Active $\alpha 5 \beta 1$
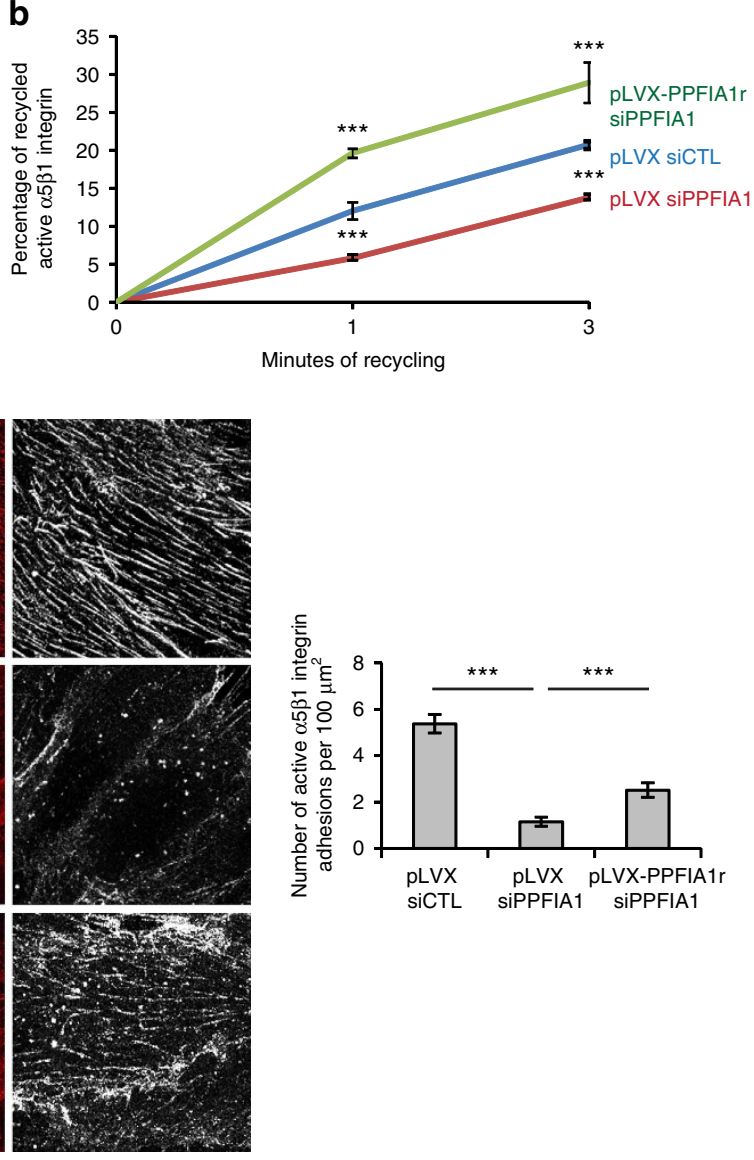
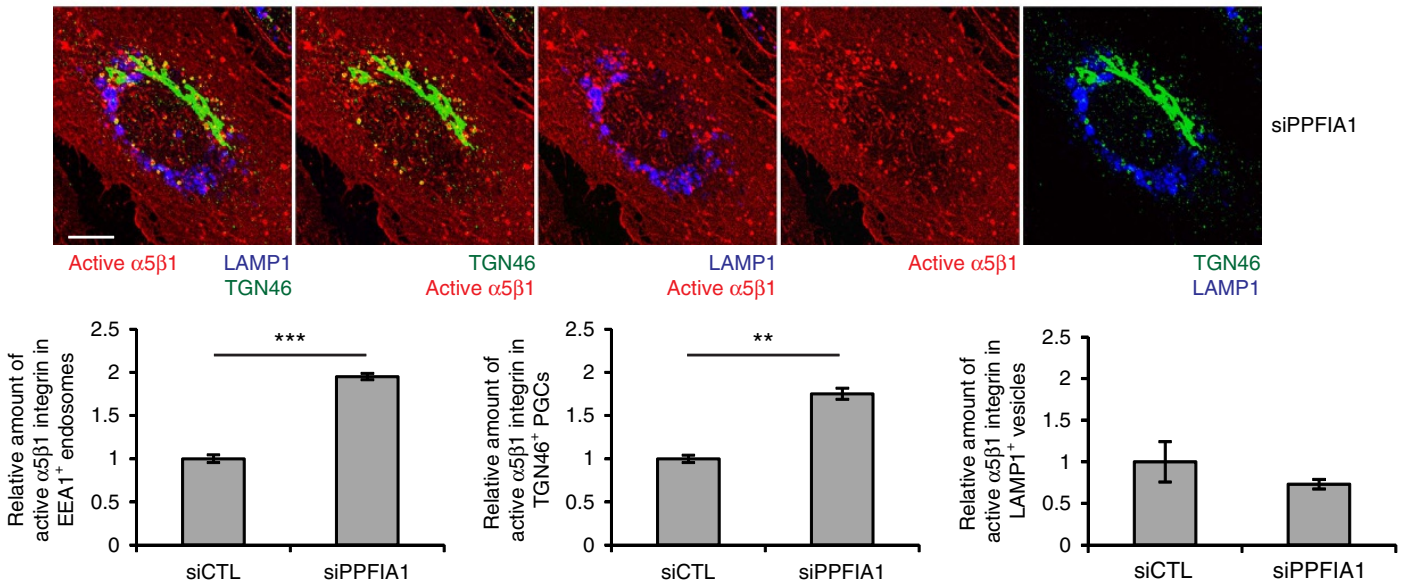
also localizes (Fig. 5a). To evaluate further the possible targeting of PGCs in close proximity $(100 \mathrm{~nm})$ to adhesion sites, we performed time-lapse total internal reflection fluorescence (TIRF) microscopy on living ECs co-transfected with the GFP-tagged TGN marker sialyltransferase (ST-GFP, gift of Julia von Blume) and the Cherry-tagged adhesion site component vinculin (Cherry-vinculin, gift of Kenneth Yamada). ST-GFP localized in typical perinuclear TGN cisternae (Fig. 5b, white arrowhead), as well as in more peripheral vesicular PGCs, several of which were observed targeting close to Cherry-vinculin-containing adhesion sites (Supplementary Movie 1; Fig. 5b; Supplementary Fig. 6a). Similarly, TIRF microscopy revealed that ST-GFP ${ }^{+}$PGCs are also delivered in close proximity to PPFIA1-Cherry-positive structures (Supplementary Movie 2; Fig. 5c; Supplementary Fig. 6b) that concentrate around vinculin-CFP-containing ECM adhesions (Fig. 1; ref. 33). Quantitative analyses of snapshots from live time-lapse TIRF microscopy unveiled in siCTL, but not in siPPFIA1 ECs, a statistically significant preferential targeting of $\mathrm{ST} \mathrm{GFP}^{+}$PGCs in proximity of Cherry-vinculin-labelled ECM adhesions, as opposed to Cherry-vinculin-devoid non-adhesive plasma membrane regions (Fig. 5d).

To sum up, when PPFIA1 is absent, endocytosed active $\alpha 5 \beta 1$ integrins accumulate in PGCs and integrin recycling to the EC surface slows down. Furthermore, on PPFIA1 silencing, a significant fraction of endogenous ED-A FN arrests in the TGN and fails to polymerize. Thus, PPFIA1 controls the recycling of internalized active $\alpha 5 \beta 1$ integrin as well as the polarized basolateral secretion and polymerization of newly synthesized endogenous ED-A FN in ECs.

Fibrillar adhesions and FN fibrils are dynamic structures. Since impairing the polarized basolateral secretion of newly synthesized ED-A FN diminishes FN fibrillogenesis (Fig. 4a), we hypothesized that the presence of the FN network underneath confluent ECs depends on its continuous turnover, which in turn hinges on a fine balance between endocytosis-mediated removal of old and cleaved FN fragments and exocytosis-mediated deposition of new FN dimers. To explore this hypothesis directly, we sought to impair the endocytosis of active $\alpha 5 \beta 1$ integrins from fibrillar adhesions and determine how this affects FN fibril formation in PPFIA1-silenced ECs. To interfere with active $\alpha 5 \beta 1$ integrin internalization, we focused on the small GTPase Rab21, which has been reported to control active $\beta 1$ integrin endocytosis ${ }^{44,45}$ and localizes both on early endosomes and at the $\mathrm{TGN}^{46}$. We found Rab21 to control the endocytosis of endogenous ED-A FN in ECs (Fig. 6a). a $\mathrm{pLVX}$ siCTL

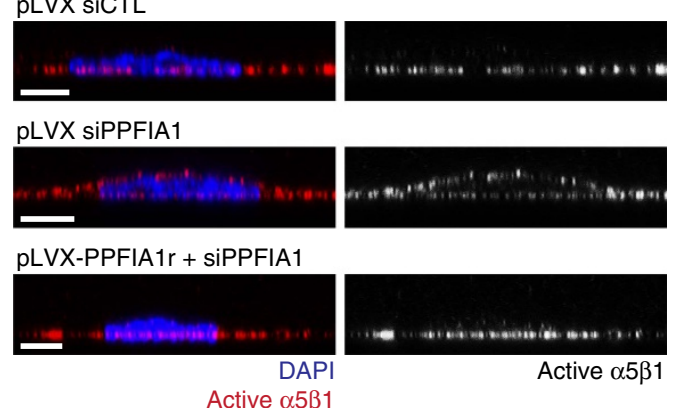

b

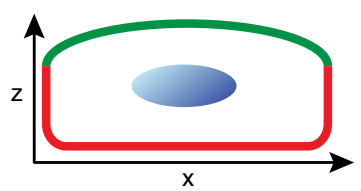

Apical fluorescence intensity/apical membrane length Basolateral fluorescence intensity/b.l. membrane length

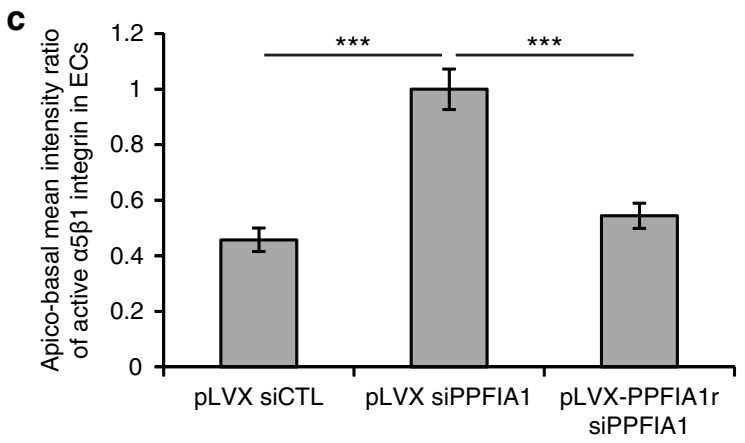

Figure 3 | PPFIA1 drives basolateral localization of active $\alpha 5 \beta 1$ integrin. (a) Confocal $x z$ sectioning microscopy analysis of anti-active $\alpha 5 \beta 1$ integrin cell surface localization (red) following 20 min of incubation with SNAKA51 $\mathrm{mAb}$ on living confluent ECs. (b) Schematic representation of the quantification of apico-basal mean intensity ratio of SNAKA51 ${ }^{+}$active $\alpha 5 \beta 1$ integrin distribution on the EC surface. b.l., basolateral.

(c) Quantitative analysis of apico-basal mean intensity ratio of SNAKA51+ active $\alpha 5 \beta 1$ integrin. SNAKA51 ${ }^{+}$active $\alpha 5 \beta 1$ integrin localizes on the basolateral surface of pLVX siCTL ECs, but not pLVX siPPFIA1 ECs in which it randomly redistributes all around the cell surface. Data are mean \pm s.e.m., $n=20$ cells per condition pooled from two independent experiments. Scale bar, $5 \mu \mathrm{m}(\mathbf{a}){ }^{\star \star \star} P<0.001$; Student's $t$-test.

Figure 2 | PPFIA1 drives the recycling of endocytosed active $\mathbf{\alpha 5 \beta 1}$ integrin. (a) Western blot analysis of EC lysates, transduced with pLVX lentivirus carrying or not the silencing-resistant PPFIA1 construct (PPFIA1r) and then control (siCTL) or PPFIA1 (siPPFIA1) silenced. (b) Time-course analysis of the relative amounts of recycled active $\alpha 5 \beta 1$ integrin in pLVX siCTL ECs versus pLVX siPPFIA1 ECs or siPPFIA1 ECs rescued with pLVX-PPFIA1r, evaluated by integrin recycling assay and capture ELISA assay. PPFIA1 silencing significantly impairs the recycling of active $\alpha 5 \beta 1$ integrin. Values are mean \pm s.e.m., $n=3$ independent experiments (two technical replicates for each experiment). (c) Confocal microscopy analysis of anti-active $\alpha 5 \beta 1$ integrin mAb SNAKA51 localization (red) in living confluent ECs following 20 min of incubation. SNAKA51+ active $\alpha 5 \beta 1$ integrin localizes in fibrillar adhesion in pLVX siCTL, but not in PLVX siPPFIA1 ECs in which it accumulates instead in perinuclear punctae. PLVX-mediated PPFIA1r overexpression restores the fibrillar adhesion localization of SNAKA51 in siPPFIA1 ECs. The number of active $\alpha 5 \beta 1$ integrin-containing adhesions per $100 \mu \mathrm{m}^{2}$ of cell area was quantified in pLVX siCTL, pLVX siPPFIA1 or pLVX-PPFIA1r + siPPFIA1 ECs. Data are mean values \pm s.e.m., $n=20$ cells per condition pooled from two independent experiments. (d) Fluorescent confocal microscopy analysis of EC perinuclear punctae in which SNAKA51 ${ }^{+}$active $\alpha 5 \beta 1$ integrin accumulates on PPFIA1 silencing. In siPPFIA1 ECs, SNAKA51 ${ }^{+}$active $\alpha 5 \beta 1$ integrin is enriched in a perinuclear vesicular compartment labelled by both TGN (TGN46) and early endosome (EEA1), but not late endosome (LAMP1) markers. Relative amount of fluorescence intensity of SNAKA51 ${ }^{+}$active $\alpha 5 \beta 1$ integrin, respectively on EEA $1^{+}$ endosomes or on TGN46 ${ }^{+}$PGCs, was evaluated. Data are mean value \pm s.e.m., $n=70$ cells per condition pooled from two independent experiments ${ }^{\star \star} P<0.01 ;{ }^{\star \star \star} P<0.001$; Student's $t$-test. Scale bar, $50 \mu \mathrm{m}(\mathbf{c}), 10 \mu \mathrm{m}(\mathbf{d})$. 
Western blots of deoxycholate (DOC) buffer-extracted insoluble ED-A FN from confluent siCTL, siPPFIA1 and siPPFIA1+ siRAB21 ECs revealed that PPFIA1 silencing dramatically reduced the amount of DOC-insoluble ED-A FN in ECs, while simultaneous silencing of PPFIA1 and Rab21 (siPPFIA1+ siRAB21) rescued the defective incorporation of endogenous
ED-A FN in the DOC-insoluble fraction of siPPFIA1 ECs (Fig. 6b). Confocal microscopy showed that, compared with siCTL ECs (Fig. 6c, top left panels), ED-A FN failed to polymerize into a fibrillar network in siPPFIA1 ECs (Fig. 6c, middle left panels), while simultaneous silencing of Rab21 GTPase $($ siPPFIA1 + siRAB21) fully restored ED-A FN polymerization in a

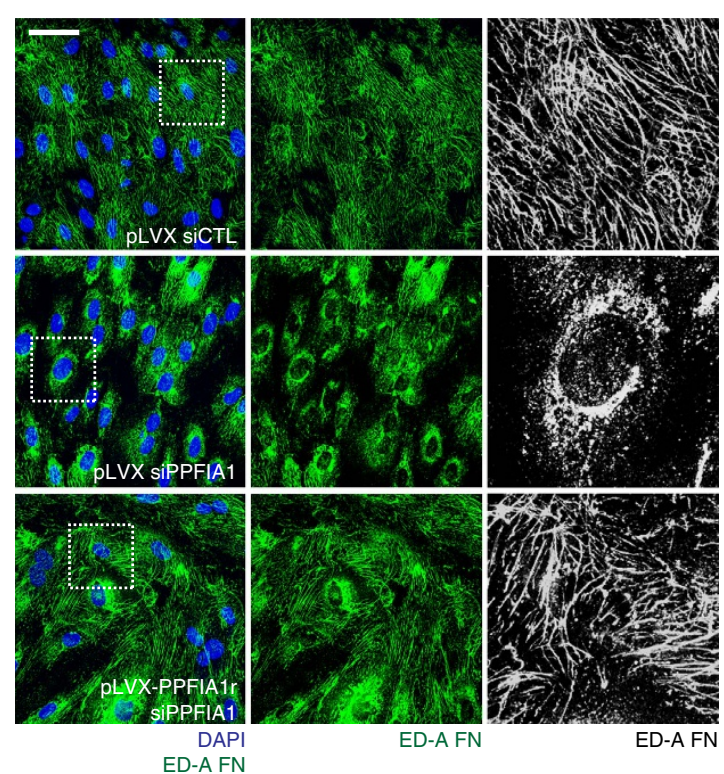

C
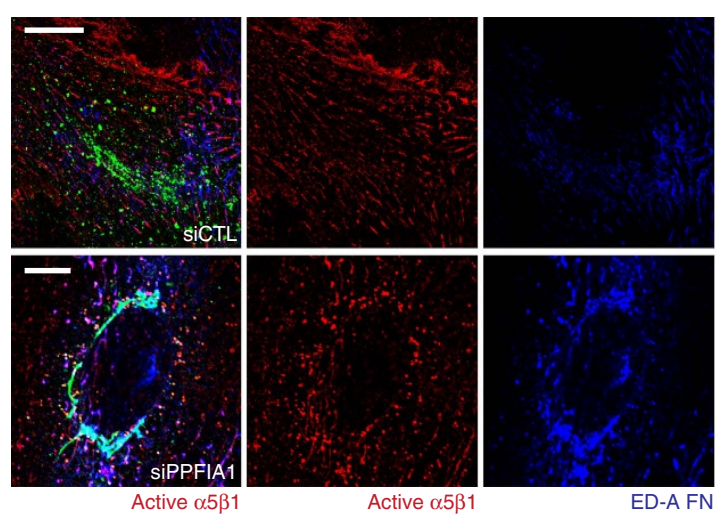

ED-A FN
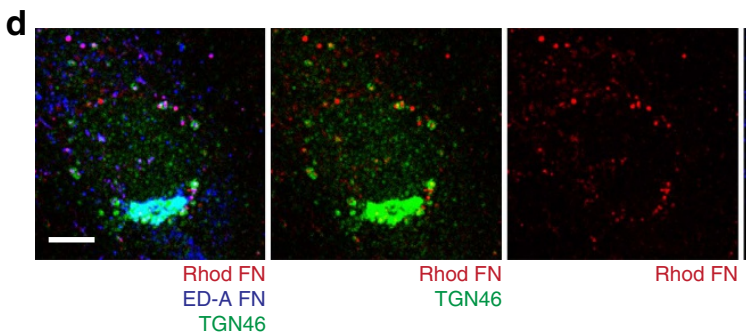

Rhod FN

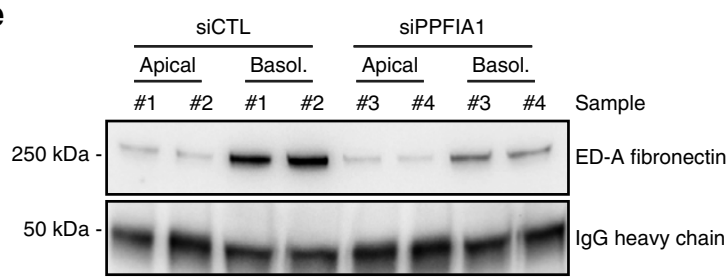

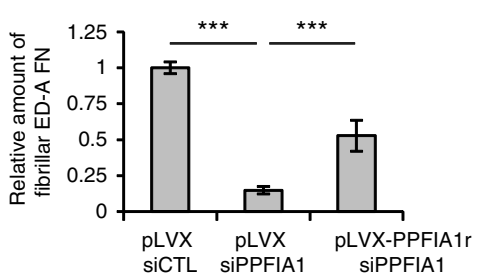

b
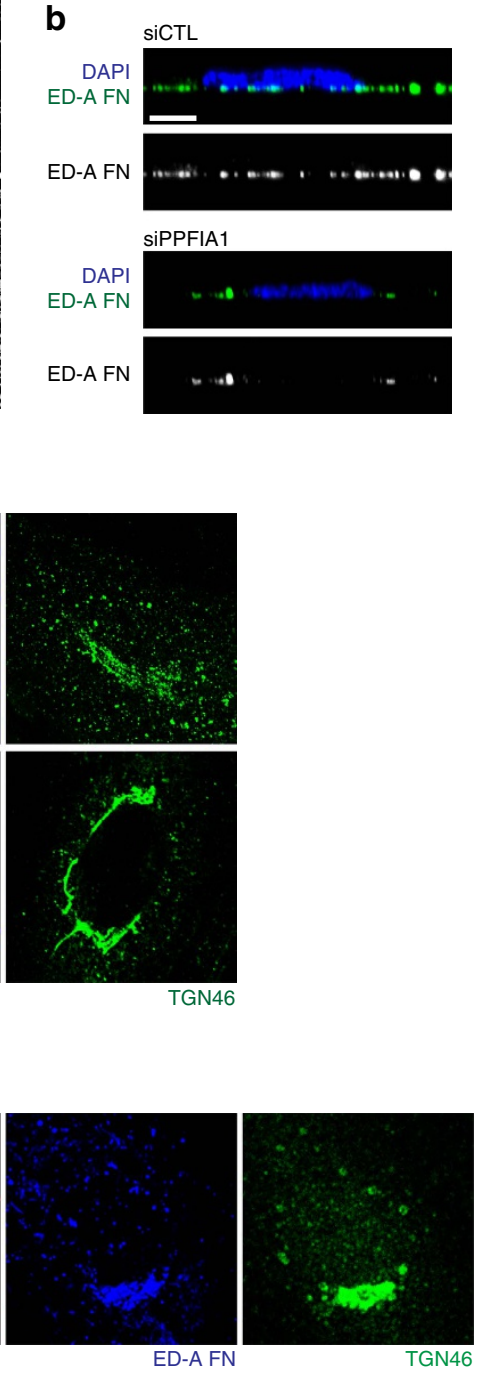

SiPPFIA1

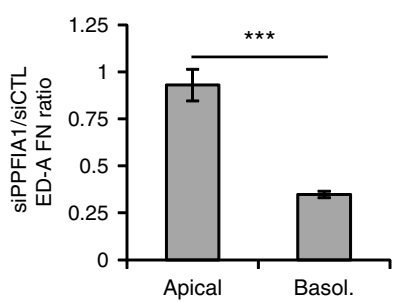


siPPFIA1 ECs (Fig. 6c, bottom left panels). Moreover, fluorescence confocal microscopy analysis revealed that, after incubation on confluent living ECs for $20 \mathrm{~min}$ at $37^{\circ} \mathrm{C}$, SNAKA51 $1^{+}$/active $\alpha 5 \beta 1$ integrin localized to fibrillar adhesion in siCTL (Fig. $6 \mathrm{c}$, top right panels), but not in siPPFIA1 ECs (Fig. 6c, middle right panels). Importantly, simultaneous PPFIA1 and Rab21 (siPPFIA1 + siRAB21) silencing reestablished the localization of SNAKA51 in fibrillar adhesions of siPPFIA1 ECs (Fig. 6c, bottom right panels).

Thus, the plasticity of fibrillar adhesions and the deposition of FN fibrils depend on the functional connection of Rab21 and PPFIA1 that respectively signal to control the endocytosis and recycling/exocytosis of active $\alpha 5 \beta 1$ integrin and cellular ED-A FN.

PI4KB/AP-1A/PTPRF-driven FN release and active $\alpha 5 \beta 1$ traffic. To identify proteins involved in the recycling of endocytosed active $\alpha 5 \beta 1$ integrin in ECs, we investigated the role of crucial regulators of PGC biogenesis ${ }^{21}$, such as phosphatidylinositol 4-kinase, catalytic, beta (PI4KB) and the EC-expressed clathrin adaptor protein complex-1A (AP-1A). In addition, we characterized the function of PTPRF that directly binds ${ }^{28}$ and cooperates with PPFIA1 in the docking of TGN-derived neurotransmitter vesicles to the active zone of neuronal presynaptic nerve terminals ${ }^{31,32}$. To this end, we examined by confocal microscopy the deposits of endogenous cellular ED-A FN in confluent ECs silenced with siCTL or a pool of four siRNA directed against either PTPRF (siPTPRF) or PI4KB (siPI4KB) (Supplementary Fig. 7). Endogenous ED-A FN polymerized into a fibrillar network in siCTL (Fig. 7a, top left panels), but neither in siPTPRF (Fig. 7a, middle left panels) nor in siPI4KB (Fig. 7a, lower left panels) ECs. Similarly, SNAKA51 ${ }^{+} /$active $\alpha 5 \beta 1$ integrin localized in fibrillar adhesion of siCTL, but not in siPTPRF or siPI4KB ECs (Fig. 7a, right panels). Quantitative analysis of apico-basal mean intensity ratio of SNAKA51 $1^{+} /$active $\alpha 5 \beta 1$ integrin by confocal $\mathrm{xz}$ sectioning on confluent ECs revealed that SNAKA $51^{+}$/active $\alpha 5 \beta 1$ integrins localized on the basolateral surface of siCTL. However, in siPPFIA1, siPTPRF, or siPI4KB ECs SNAKA51 $1^{+}$/active $\alpha 5 \beta 1$ integrins redistributed randomly all around the cell surface (Fig. 7b). For control purposes, we silenced the liprin $\beta$ protein PPFIBP1, that similarly to PPFIA1 binds PTPRF and control synaptic size ${ }^{30}$, but whose function in organizing the traffic of presynaptic nerve terminals is poorly understood ${ }^{29}$. We found that PPFIBP1 silencing only modestly affected the apico-basal distribution of SNAKA $51^{+}$/ active $\alpha 5 \beta 1$ integrin in ECs (Fig. 7b). Furthermore, silencing of either PI4KB (Fig. 7c) or the key AP-1A subunit AP1M1 (ref. 47)
(siAP1M1) (Fig. 7d) or PTPRF (Fig. 7e) significantly impaired the recycling of SNAKA51 $1^{+}$/active $\alpha 5 \beta 1$ integrin. As in the case of active $\alpha 5 \beta 1$ integrin apico-basal distribution, knocking down PPFIBP1 affected the recycling of SNAKA51 ${ }^{+} /$active $\alpha 5 \beta 1$ integrin much less efficiently (Fig. $7 \mathrm{f}$ ).

Altogether, these data indicate that, similarly to PPFIA1 (Fig. 4), PI4KB, AP-1A and PTPRF control the recycling of active $\alpha 5 \beta 1$ integrin as well as the polymerization of a FN fibrillar network underneath cultured ECs. Furthermore, the data show that the internalized active $\alpha 5 \beta 1$ integrins exploit the same $\mathrm{PI} 4 \mathrm{~KB} / \mathrm{AP}-1 \mathrm{~A}-$ dependent trafficking pathway for recycling.

Rab11B drives FN secretion and active $\alpha 5 \beta 1$ recycling. The results above indicated how in ECs, the same molecular and cellular mechanisms control active $\alpha 5 \beta 1$ integrin recycling as well as the basolateral secretion and polymerization of ED-A FN. Rab11 small GTPases, which localize at recycling endosomes, TGN and $\mathrm{PGCs}^{48}$, regulate the slow recycling of active $\beta 1$ integrins ${ }^{13,39}$. Next, we investigated if in ECs Rab11 proteins may simultaneously orchestrate active $\alpha 5 \beta 1$ integrin recycling together with ED-A FN secretion and fibrillogenesis. We found that ECs express both Rab11A and Rab11B proteins (Supplementary Fig. 7). In polarized epithelial cells, Rab11A resides in a vesicular compartment distinct from that of $\mathrm{Rab}_{11 \mathrm{~B}}{ }^{49}$ and, Rab11A, but not Rab11B, controls the apical delivery of postGolgi cargoes ${ }^{50}$. When compared with siCTL ECs, Rab11B (siRab11B), but not Rab11A (siRab11A) knockdown halved the amount of recycled active $\alpha 5 \beta 1$ integrin in ECs (Fig. 8a). Similarly, on incubation on living confluent ECs, SNAKA51 mAb largely localized in $\alpha 5 \beta 1$-containing fibrillar adhesions of siCTL and siRab11A, but not siRab11B ECs (Fig. 8b). In addition, quantitative confocal $\mathrm{xz}$ sectioning analysis on confluent ECs revealed that although SNAKA51 ${ }^{+}$/active $\alpha 5 \beta 1$ integrins localize along the basolateral side of siCTL and siRab11A ECs, they redistributed randomly around the surface of siRab11B ECs (Fig. 8c). In addition, while siCTL and siRab11A ECs polymerized ED-A FN into an extracellular fibrillar network, siRab11B ECs accumulated $\mathrm{FN}$ in a perinuclear compartment, consisting of TGN46 ${ }^{+}$TGN cisternae (Fig. 8d). Moreover, in siRab11B ECs SNAKA $51^{+} /$active $\alpha 5 \beta 1$ integrins accumulated in perinuclear $\mathrm{TGN}^{+}{ }^{+}$PGCs (Fig. 8d). Finally, quantitative biochemical apico-basal secretion assays revealed that Rab11B silencing preferentially impaired basolateral ED-A FN secretion in the medium of confluent cultured ECs (Fig. 8e). We hence infer that in ECs the Rab11B small GTPase orchestrates the recycling of endocytosed active $\alpha 5 \beta 1$ integrins together with the basolaterallypolarized secretion and polymerization of endogenous ED-A FN.

Figure 4 | PPFIA1 controls endogenous ED-A FN exit from the TGN and its polymerization underneath confluent ECs. (a) Confocal microscopy analysis of IST9 mAb-labelled endogenous ED-A FN (green) in confluent ECs. ED-A FN polymerizes into a fibrillar network in pLVX siCTL, but not in pLVX siPPFIA1 ECs in which it accumulates in perinuclear punctae. PLVX-mediated PPFIA1r overexpression restores ED-A FN polymerization in siPPFIA1 ECs. The relative amount of fibrillar ED-A FN area was calculated in pLVX siCTL, pLVX siPPFIA1 and pLVX-PPFIA1r + siPPFIA1 ECs. Data are mean \pm s.e.m., $n=20$ cells per condition pooled from two independent experiments. (b) Confocal xz sections of ED-A FN (green) in siCTL or siPPFIA1 ECs. Compared with siCTL ECs, the basolateral accumulation of polymerized ED-A FN decreases dramatically in siPPFIA1 ECs. (c) Confocal microscopy characterization of the perinuclear compartment in which ED-A FN accumulates on PPFIA1 silencing in ECs. Before fixation, living ECs were incubated for 20 min with exogenous SNAKA51. Compared with siCTL ECs, ED-A FN (blue) heavily accumulates in the TGN46+ (green) TGN cisternae of siPPFIA1 ECs. Of note, in siPPFIA1 ECs exogenously added SNAKA51 mAb (red) binds active $\alpha 5 \beta 1$ integrins that, on endocytosis, reach TGN46+ post-Golgi carrier vesicles, but do not enter in TGN cisternae. (d) Fluorescent confocal microscopy shows that, similarly to SNAKA51, exogenously added rhodamine (Rhod, red) labelled serum FN reaches TGN46+ (green) post-Golgi carrier vesicles, but does not enter in TGN cisternae of siPPFIA1 ECs in which endogenous ED-A FN (blue) heavily accumulates instead. (e) Western blot analysis of soluble ED-A FN released by confluent ECs seeded on Transwell inserts. An equal percentage of apical and basolateral volumes of medium were collected after $72 \mathrm{~h}$ of culture from different wells of siCTL or siPPFIA1 ECs. Equal amounts of exogenous rabbit IgG were added to samples (spike normalization) for loading control purposes. Quantification of the ratio between apical or basolateral amount of ED-A FN released by siCTL over siPPFIA1 ECs. PPFIA1 silencing impairs basolateral, but not apical ED-A FN secretion. Data are mean \pm s.e.m., $n=8$ wells per condition pooled from 4 independent experiments. Scale bar, $50 \mu \mathrm{m}(\mathbf{a}), 10 \mu \mathrm{m}(\mathbf{c}, \mathbf{d}), 5 \mu \mathrm{m}(\mathbf{b}) .{ }^{\star \star \star} P<0.001$; Student's $t$-test. 
$\alpha 5 \beta 1$ controls FN secretion and polymerization. Altogether, the above results led us to postulate that $\alpha 5 \beta 1$ integrin may play a key direct role in the control of ED-A FN secretion. To verify this hypothesis, we investigated whether interfering with $\alpha 5 \beta 1$ integrin function in ECs may indeed hamper the secretion of
ED-A FN from the TGN and its assembly into a basolateral fibrillar network. Therefore, to hinder directly $\alpha 5 \beta 1$ integrin function, we silenced the $\alpha 5$ integrin subunit (siITGA5) in ECs (Fig. 9a). Of note, in addition to the expected severe impairment of FN fibrillogenesis, the silencing of $\alpha 5$ integrin subunit also
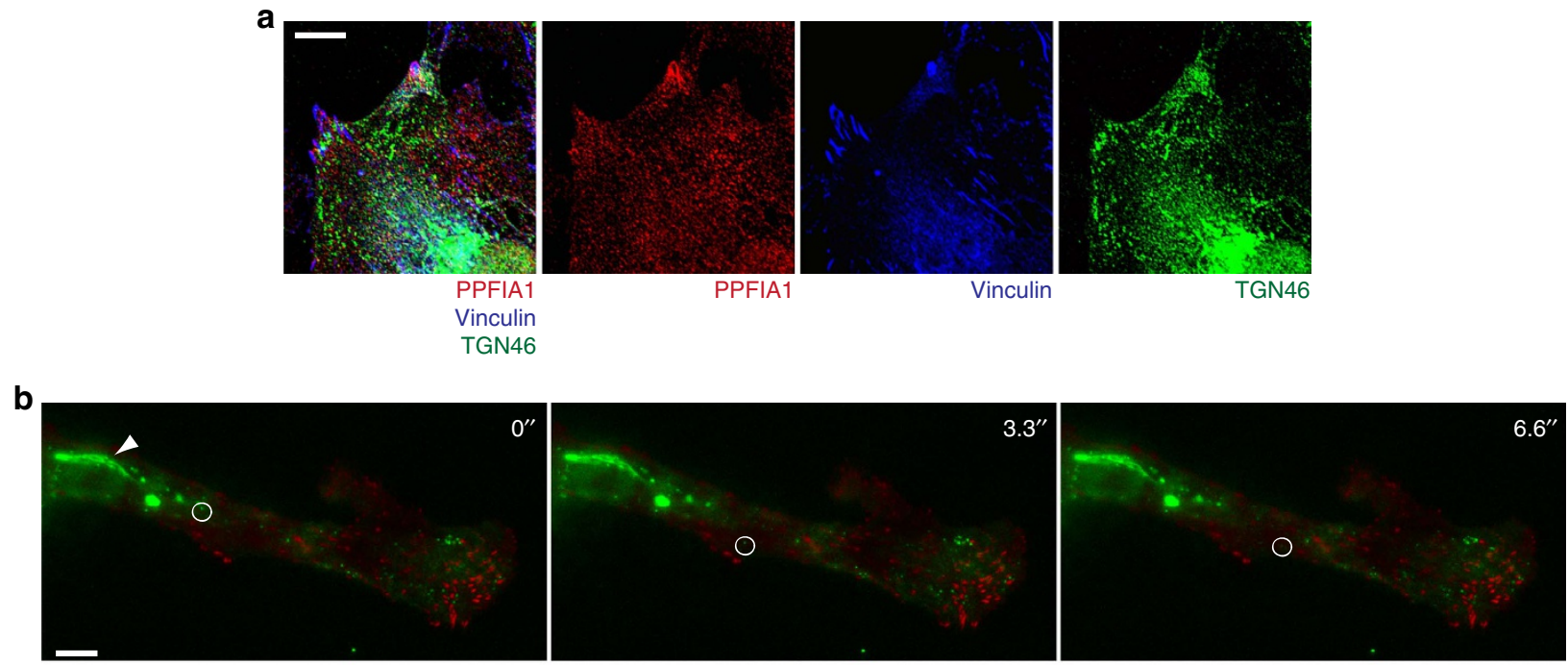
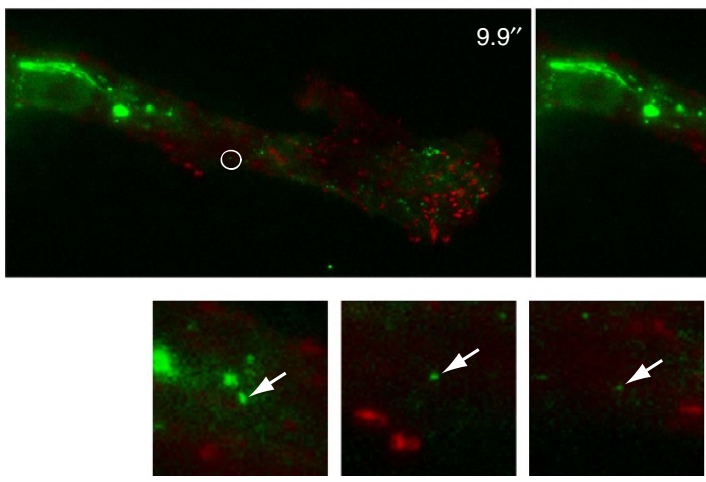

$6.6^{\prime \prime}$

$0^{\prime \prime}$

$3.3^{\prime \prime}$
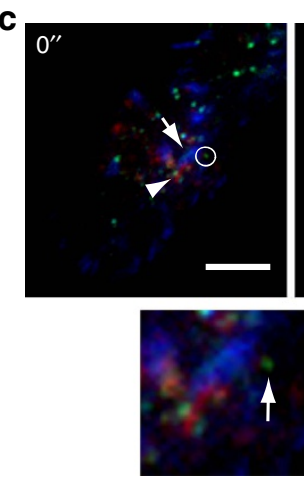

$0^{\prime \prime}$
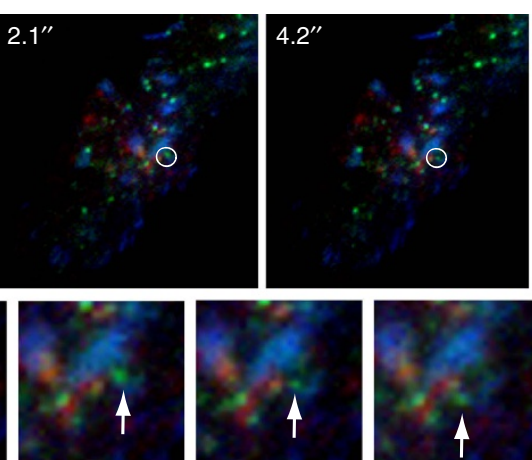

$2.1^{\prime \prime}$

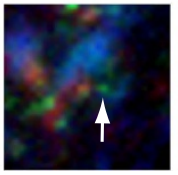

$4.2^{\prime \prime}$ $6.3^{\prime \prime}$

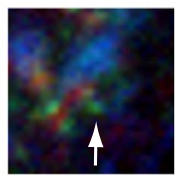

d

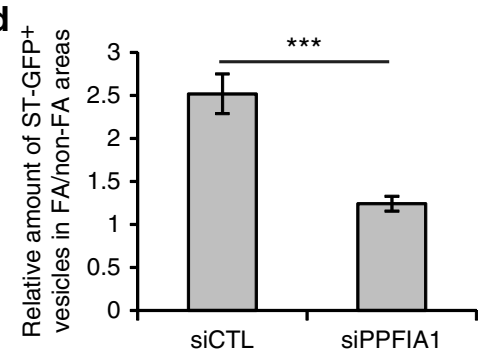

PPFIA1-Cherry

ST-GFP

Vinculin-CFP 
promoted a clear accumulation of endogenous ED-A FN in the TGN of ECs (Fig. 9b). Furthermore, quantitative biochemical apico-basal secretion assays unveiled that $\alpha 5$ integrin subunit silencing significantly decreases the basolateral, but not apical secretion of ED-A FN in the medium of confluent cultured ECs (Fig. 9c). Thus, blocking $\alpha 5 \beta 1$ integrin function in ECs inhibits the basolateral secretion of endogenous ED-A FN from the TGN.

PPFIA1 drives vascular morphogenesis in vitro and in vivo. Polarized FN secretion and matrix polymerization are required for vascular morphogenesis in vitro ${ }^{7}$ and for vessels formation in vivo in the developing mouse embryo ${ }^{1}$. First, we directly tested the influence of PPFIA1 on vascular morphogenesis in vitro by means of EC capillary formation assays in growth factor-reduced Matrigel. After $8 \mathrm{~h}$ incubation at $37^{\circ} \mathrm{C}$, pLVX siCTL ECs formed capillary networks surrounded by a dense meshwork of polymerized cellular ED-A FN. By contrast, pLVX siPPFIA1 ECs failed to form a fibrillar FN network and remained immobile on the Matrigel surface without forming cell-to-cell contacts and a capillary network. Importantly, pLVX-mediated PPFIA1r re-expression rescued FN network formation, EC mobility and the formation of capillary networks (Supplementary Fig. 8).

Next, we assessed the effects of PPFIA1 inactivation in vivo. Antisense morpholino oligonucleotides (MOs) were used to knockdown Ppfial protein expression in the transgenic zebrafish line $T g(k d r l: E G F P)$ expressing EGFP in ECs. To this end, a translation blocking MOs (experimental group; MO-ppfia1) and the corresponding five-base mismatch MOs (control group; MO-CTL) were independently microinjected into fertilized zebrafish eggs at the single-cell stage. At $48 \mathrm{~h}$ post-injection (hpi), the gross appearance of ppfial morphant embryos was normal. However, approximately $35 \%$ of them exhibited cardiovascular defects, such as an enlarged heart chamber phenotype due to atrium dilation associated with pericardial oedema, blood stasis (Fig. 10a), and reduced blood flow despite the presence of cardiac activity (Supplementary Movies 3 and 4). In contrast, control-injected embryos appeared phenotypically normal. Furthermore, sporadic haemorrhages in the subintestinal vascular plexus, malformations in the intersegmental vessels (Se) and irregular shapes (margin) of the dorsal aorta (DA) and the posterior cardinal vein (PCV) were also present in defective ppfial morphants (Fig. 10a). At $72 \mathrm{hpi}$, all these phenotypic cardiovascular alterations persisted, but did not further exacerbate.

To exclude the possibility that cardiovascular aberrations of ppfial morphants might be due to MO off-target effects, we assessed whether co-injecting human PPFIA1 mRNA, which was not targeted by either ppfial MOs, could rescue the observed phenotypes ${ }^{51}$. We analyzed wild type embryos, ppfial morphants and ppfial morphants co-injected with human PPFIA1 mRNA, confirming its effective translation into the corresponding
PPFIA1 protein by western blot, although at lower levels than that of endogenous Ppfial in wild type embryos (Fig. 10b). As previously observed, about $35 \%$ of $48 \mathrm{hpi}$ ppfial morphants exhibited the described cardiovascular defects, while controlinjected embryos were unaffected. Human PPFIA1 mRNA co-injection successfully rescued the cardiovascular defects in the large majority $(\sim 84 \%)$ of ppfial morphants that appeared morphologically normal (Fig. 10a,c), while a minor fraction of them $(\sim 16 \%)$ still exhibited cardiovascular abnormalities.

Next, we directly evaluated whether the lack of Ppfial may affect the deposition of FN in the living zebrafish. To this end, we performed quantitative fluorescence confocal microscopy analyses of $\mathrm{FN}$-immunostained trunk sections of $72 \mathrm{hpi}$ wild type embryos, ppfial morphants and ppfial morphants co-injected with human PPFIA1 mRNA. As shown in Fig. 10d, ppfial morphants exhibited a sizeable reduction in FN deposition both around DA and PCV and in overall trunk sections (Fig. 10d). Co-injection of human PPFIA1 mRNA in ppfial morphants rescued FN deposition defects albeit not completely (Fig. 10d), suggesting a dose-dependent threshold for the different phenotypic alterations observed in ppfial morphants (Fig. 10b).

In summary, PPFIA1 depletion inhibits endothelial FN secretion and polymerization, and impairs vascular morphogenesis in cultured ECs and in the developing zebrafish embryo.

\section{Discussion}

The onset of cell polarity is a key event for organ development ${ }^{52}$ in multicellular organisms ${ }^{53}$. Polarized cells of epithelial tissues, nervous and vascular systems and invading cancers, are characterized by spatially and functionally defined membrane areas that originate from, and are maintained by, complexes of molecules dedicated either to the compartmentalization or to the directional targeting of their own structural components ${ }^{22}$. Once established, cells must coordinate the maintenance of polarity with the continuous turnover of transmembrane proteins and ECM at specific subcellular sites. In contrast to fibroblasts ${ }^{42}$, FN deposition is for example restricted to the basolateral surface of polarized ECs ${ }^{17}$.

In the case of epithelial cells $\mathrm{s}^{22}$ and $\mathrm{ECs}^{5}$, polarization is defined by an apical and a basolateral plasma membrane area. It has been proposed that $\beta 1$ integrins act as sensors that define and orient the apico-basal cell axis ${ }^{4-6}$. During embryonic cardiovascular development, basolateral FN secretion and assembly into a bioactive fibrillar network by EC-expressed $\beta 1$ integrins are crucial for the establishment of endothelial apico-basal polarity and vascular morphogenesis ${ }^{3}$. Hence, $\beta 1$ integrins may define the orientation of the apico-basal axis and at the same time convey positional information, allowing a continuous basally polarized secretion of newly synthesized FN and giving rise to a selfsustaining positive feedback loop. Here, we have investigated this possibility. We found that in ECs the cytoplasmic adaptor

Figure 5 | Post-Golgi carrier vesicles target EC adhesion sites. (a) Confocal microscopy analysis on fixed ECs shows that, in addition to labelling the perinuclear TGN cisternae, TGN46 (green) is present in post-Golgi carrier vesicles that preferentially accumulate, together with PPFIA1 (red), around vinculin-containing (blue) adhesion sites. (b) Snapshots from live TIRF microscopy of a region of an EC that was co-transfected with the TGN marker ST-GFP (green) and the adhesion site component Cherry-vinculin (red). ST-GFP is clearly localized at the TGN cisternae (white arrowhead) and in peripheral post-Golgi vesicles, the trajectory of one of which (white circle) is tracked from the perinuclear area to a peripheral Cherry-vinculin-labelled adhesion site. Single channel photograms are shown in Supplementary Fig. 3. (c) Snapshots from live TIRF microscopy of a region of an EC that was co-transfected with ST-GFP (green), PPFIA1-Cherry (red) and the vinculin-CFP (blue). The trajectory of one ST-GFP post-Golgi vesicles (white circle) is tracked in proximity of a CFP-vinculin labelled adhesion site (white arrow), where it reaches a PPFIA1-Cherry ${ }^{+}$area (white arrowhead) and resides for at least $12 \mathrm{~s}$. More in general, several ST-GFP-labelled vesicles closely associate to PPFIA1 surrounded vinculin-containing adhesion sites (Supplementary Movies 1 and 2). Single channel photograms are shown in Supplementary Fig. 3. (b,c) Lower panels are magnifications of the white circled areas shown in the above panels. White arrows indicate representative ST-GFP + PGCs that are reaching PPFIA1-Cherry ${ }^{+}$areas. (d) Quantification of the relative amount of ST-GFP vesicles residing in focal adhesion containing (FA) or non-containing (non-FA) areas in siCTL or siPPFIA1 ECs. Data are mean \pm s.e.m., $n=10$ cells per condition pooled from three independent experiments. Scale bar, $10 \mu \mathrm{m}(\mathbf{a}-\mathbf{c})$. ${ }^{\star \star \star} P<0.001$; Student's $t$-test. 

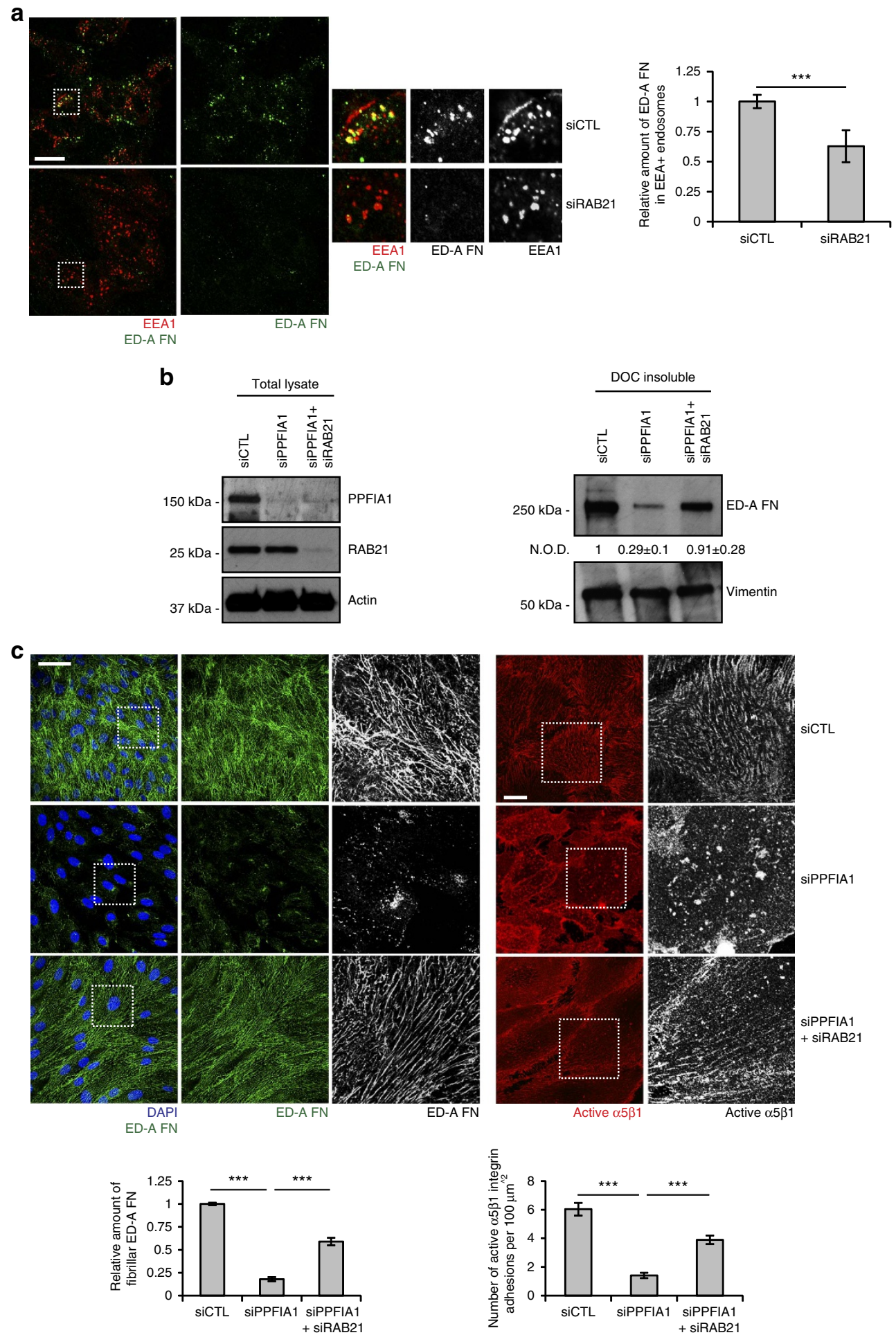

Figure 6 | Fibrillar adhesions and FN fibrils are dynamic structures governed by Rab21 and PPFIA1. (a) Living siCTL and siRAB21 ECs were incubated with IST9 mAb for $30 \mathrm{~min}$, fixed, acid washed and stained. RAB21 silencing impairs ED-A FN endocytosis as revealed by the decrease of IST9 punctae co-localizing with the endosome marker EEA1. Right panels are magnifications of the boxed areas in left panels. Data are mean value \pm s.e.m., $n=70$ cells per condition pooled from two independent experiments. ${ }^{\star \star \star} P<0.001$. (b) Left panel, western blot analysis of PPFIA1, RAB21 and actin on total lysates of siCTL, siPPFIA1 and siPPFIA1 + siRAB21 ECs. Right panel, western blot analysis of the insoluble matrix fraction of ECs that were extracted with DOC buffer. PPFIA1 silencing dramatically reduces the amount of DOC-insoluble fraction of endogenous ED-A FN. Of note, simultaneous silencing of Rab21 GTPase (siPPFIA1 + siRAB21), which drives integrin endocytosis, rescues the defective incorporation of endogenous ED-A FN in the DOC-insoluble fraction of siPPFIA1 ECs. (c) Confocal microscopy analysis of the patterning of endogenous cellular ED-A FN (green) in fixed confluent ECs. Before fixation, living ECs were incubated for 20 min with exogenous SNAKA51 (red). ED-A FN polymerizes into a fibrillar network in siCTL, but not in siPPFIA1 ECs. Simultaneous silencing of Rab21 GTPase (siPPFIA1 + siRAB21) fully restores ED-A FN polymerization in siPPFIA1 ECs. SNAKA51 ${ }^{+}$active $\alpha 5 \beta 1$ integrin localizes in fibrillar adhesion in siCTL, but not in siPPFIA1 ECs. Notably, simultaneous Rab21 (siPPFIA1 + siRAB21) silencing promotes the localization of SNAKA51 in fibrillar adhesion of siPPFIA1 ECs. Data are mean \pm s.e.m., $n=20$ cells per condition pooled from two independent experiments. Scale bar, $20 \mu \mathrm{m}$ (a,c, right), $50 \mu \mathrm{m}$ (c, left). ${ }^{\star \star \star} P<0.001$; Student's $t$-test. 
PPFIA1: (i) localizes close to $\alpha 5 \beta 1$-containing fibrillar adhesions; (ii) physically interacts with the cytodomain of active $\alpha 5 \beta 1$ integrin; (iii) controls the recycling to the cell surface of endocytosed active $\alpha 5 \beta 1$ integrin; (iv) drives the polarized distribution of active $\alpha 5 \beta 1$ integrin on the basolateral cell surface; (v) promotes the localization of PGCs near ECM adhesions. These observations, together with the fact that, on PPFIA1 silencing, active $\alpha 5 \beta 1$ integrin accumulates in PGCs, support a working model in which PPFIA1 allows the polarized recycling of active $\alpha 5 \beta 1$ integrin to fibrillar adhesions of the

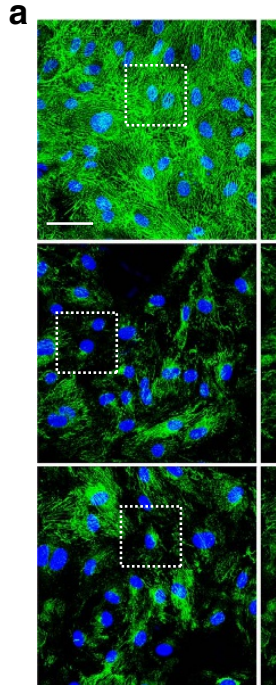

ED-A FN/DAP

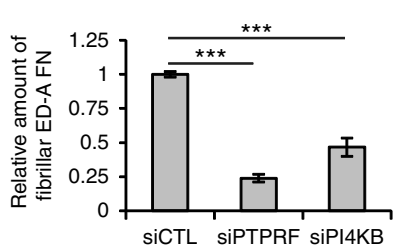

b
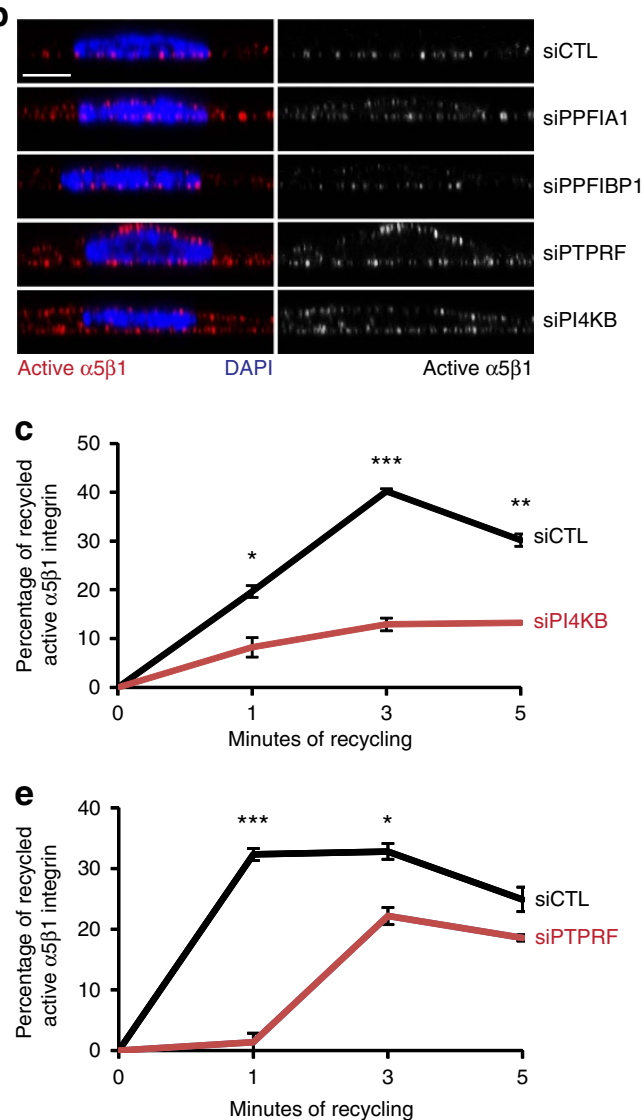
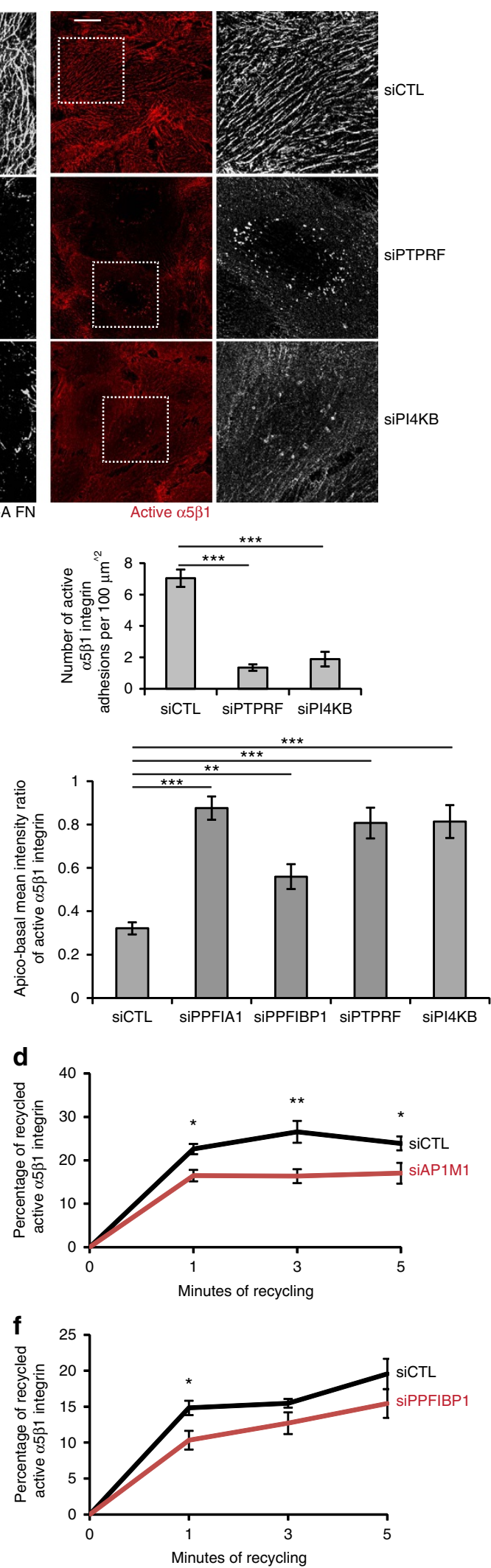
basolateral side of ECs by exploiting TGN-mediated secretory pathways and interacting with $\beta 1$ integrin cytotails. On internalization into early endosomes, active $\alpha 5 \beta 1$ integrins, likely bound to protease-cleaved fragments of polymeric extracellular $\mathrm{FN}^{9,10}$, reach PGCs from where they may recycle to the EC basolateral surface along with newly synthesized FN dimers derived from the TGN cisternae (Supplementary Fig. 9). This cellular pathway represents an ideal strategy to orchestrate the removal of endocytosed and old FN with the deposition of newly synthesized FN allowing a continuous, but dynamic renewal of polarized FN fibrils. It is still unclear how and where the old, endocytosed FN fragments separate from active $\alpha 5 \beta 1$ integrins, and if the subpopulation of new FN dimers bind active $\alpha 5 \beta 1$ integrins in PGCs or if the recycling of endocytosed active $\alpha 5 \beta 1$ integrin is just providing a positional signal for the polarized FN secretion. Since integrins are bidirectional allosteric adhesion receptors ${ }^{54}$, in the absence of sizeable mechanical tension, freshly synthesized FN binding may be sufficient to maintain $\alpha 5 \beta 1$ integrin in active conformation within PGCs, around which we did not detect talin or kindlins. In any event, our observation that the silencing of $\alpha 5$ integrin subunit in ECs impairs the basolateral secretion of endogenous ED-A FN from the TGN supports the assumption that $\alpha 5 \beta 1$ integrin controls polarized FN exocytosis.

Central players in the turnover of active $\alpha 5 \beta 1$ integrin at EC fibrillar adhesion sites are PPFIA1 and PTPRF, which may provide the information to direct $\alpha 5 \beta 1$ integrin-containing carriers for basolateral integrin recycling, while remaining at the basal surface, where they probably define specific membrane regions. Interestingly, PTPRF and PPFIA1 exert a similar function in neuronal presynaptic areas where they compartmentalize structural components of the so-called active zone and mediate neurotransmitter vesicle docking ${ }^{31,32}$. PPFIA1 is also part of a multi-protein complex that localizes close to integrin adhesion sites to control cortical microtubule dynamics and targeting ${ }^{33}$. Since microtubules are essential for polarized protein and lipid traffic ${ }^{55}$, the microtubule-related function of PPFIA1 may represent an additional and complementary strategy by which it may favour basolateral targeting of PGCs containing recycling active $\alpha 5 \beta 1$ integrins and newly synthesized FN in ECs. Further work will determine if PPFIA1 triggers additional biochemical signal(s) promoting the basolateral secretion of freshly synthesized FN.

Accumulating evidence supports the notion that integrins and their ligands exhibit a high traffic-dependent turnover within adhesion sites ${ }^{11-13}$. Small GTPases belonging to the Rab5 subfamily, namely Rab5 and Rab21, control $\beta 1$ integrin internalization $44,45,56$. Analogous to observations in fibroblasts ${ }^{56}$, we also found that endothelial fibrillar adhesions, which are considered the most mature $\alpha 5 \beta 1$ integrin-containing adhesive contacts $^{57}$, are highly dynamic in nature. However, the turnover of fibrillar adhesions in fibroblasts does not appear to be significantly influenced by the master regulator of early endocytosis Rab5 (ref. 56). Instead, we propose a model where active $\alpha 5 \beta 1$ integrin-containing fibrillar adhesions of ECs and associated FN fibrils rely on the coupling of Rab21-dependent internalization and PPFIA1-driven recycling or exocytosis from the TGN of their adhesive receptor or ligand components respectively (Supplementary Fig. 9).

The role that regulators of PGC biogenesis, such as PI4KB and AP-1 (refs $20-22$ ), play in active $\alpha 5 \beta 1$ integrin recycling suggests that, through the TGN, the biosynthetic routes connect to the endosomal paths that traffic these ECM receptors back and forth from adhesion sites. In this regard, it will be important to determine in the future whether receptors other than integrins may exploit trafficking to the TGN to convey signals modulating the biosynthetic routes according to the presence, amount and location of extracellular cues in polarized cells (Supplementary Fig. 9). In this respect, along the biosynthetic routes of epithelial cells, transmembrane proteins can be either directly routed from the TGN to the plasma membrane or trafficked through recycling endosomal compartments before reaching the apical or basolateral surface 22 . However, it is unknown if recycling endosomal compartment may be also involved in the polarized secretion of soluble proteins, such as FN. Here, we reveal how in ECs the regulator of slow endosomal recycling Rab11B coordinates with inducers of PGC formation, that is AP1M1 and PI4KB, and the plasma membrane-associated vesicle tethering complex PTPRF/PPFIA1 to control the basolateral secretion of endogenous ED-A FN in conjunction with the recycling of endocytosed active $\alpha 5 \beta 1$ integrins (Supplementary Fig. 9).

FN is required for developmental blood vessel ${ }^{1,3}$ and heart morphogenesis both in mice ${ }^{3}$ and in zebrafish ${ }^{58}$. Indeed, FN and its major receptor $\alpha 5 \beta 1$ integrin regulate blood vessel lumen formation $^{4,7}$ and the acquisition of a polarized epithelial organization by migrating myocardial progenitors ${ }^{58,59}$. The depletion of ppfial in vivo in the developing zebrafish embryo caused severe heart morphogenesis defects resembling those observed in nat $/ f n$ mutants ${ }^{58}$, impairs perivascular FN deposition, and the patterning of trunk large vessels. This data, together with the defects in EC migration, vessel formation and FN polymerization in vitro emphasizes the relevance of the molecular network capable of coordinating polarized active $\alpha 5 \beta 1$ integrin recycling and FN exocytosis in cardiovascular cells.

\section{Methods \\ Antibodies and reagents. Rabbit polyclonal anti-PPFIA1 was from Proteintech Group, Chicago, IL, USA (characterized on siCTL and siPPFIA1 ECs in Supplementary Fig. 10). Goat polyclonal anti-Nrp1 (C-19), mouse monoclonal anti-ED-A FN (clone IST9) and goat polyclonal anti-EEA1 (N-19) were from Santa Cruz Biotechnology, Dallas, TX, USA. Rabbit polyclonal anti- $\alpha 5$-integrin}

Figure 7 | PTPRF cooperates with PI4KB and AP-1A to control ED-A FN exit from the TGN and polymerization as well as active $\alpha 5 \beta 1$ integrin recycling. (a) Confocal microscopy analysis of the patterning of endogenous cellular ED-A FN (green) in fixed confluent ECs and anti-active $\alpha 5 \beta 1$ integrin mAb SNAKA51 (red) incubated on living confluent ECs for 20 min before fixation. ED-A FN polymerizes into a fibrillar network in siCTL, but neither in siPI4KB nor in siPTPRF ECs. SNAKA51 ${ }^{+}$active $\alpha 5 \beta 1$ integrin localizes in fibrillary adhesion in siCTL, but neither in siPI4KB nor in siPTPRF ECs. Relative amount of fibrillary ED-A FN area was measured in siCTL, siPTPRF and siPI4KB ECs. Data are mean \pm s.e.m., $n=20$ cells per condition pooled from two independent experiments. The number of active $\alpha 5 \beta 1$ integrin-containing adhesions per $100 \mu \mathrm{m}^{2}$ of cell area was quantified in siCTL, siPTPRF and siPI4KB ECs. Data are mean \pm s.e.m., $n=20$ cells per condition pooled from two independent experiments. (b) Quantitative analysis of apico-basal mean intensity ratio of SNAKA51 ${ }^{+}$active $\alpha 5 \beta 1$ integrin by confocal $x z$ sectioning on confluent ECs. SNAKA51 ${ }^{+}$active $\alpha 5 \beta 1$ integrin localizes on the basolateral surface of siCTL, but neither siPPFIA1, nor siPTPRF, nor siPI4KB ECs in which it randomly redistributes all around the cell surface. Silencing of PPFIBP1 affects the apico-basal distribution of SNAKA51 ${ }^{+}$active $\alpha 5 \beta 1$ integrin much less efficiently. (c-f) Time-course analysis of the relative amounts of recycled active $\alpha 5 \beta 1$ integrin in siCTL ECs versus siPPFIBP1 or siPTPRF or siAP1M1 or siPI4KB ECs, as evaluated by integrin recycling assay and capture ELISA assay. Silencing of either PTPRF or PI4KB or AP1M1 significantly impairs the recycling of SNAKA51 ${ }^{+}$active $\alpha 5 \beta 1$ integrin. Data are mean \pm s.e.m., $n=3$ independent experiments (two technical replicates for each experiment). Scale bar, $50 \mu \mathrm{m}$ (a, left), $20 \mu \mathrm{m}$ (a, right), $5 \mu \mathrm{m}(\mathbf{b}) .{ }^{\star} P<0.05 ;{ }^{\star \star} P<0.01$; ${ }^{\star \star \star} P<0.001 ;$ Student's $t$-test. 
(AB1949), mouse monoclonal anti-kindlin-2 (Mab2617) and mouse monoclonal anti-Myc (05-419) were from Merk Millipore, Billerica, MA, USA. Mouse monoclonal anti-vinculin (V9131), anti-Flag-M2-HRP (A8592) and anti- $\alpha$ tubulin (T5168) were from Sigma Aldrich, St Louis, MO, USA. Mouse monoclonal anti-actin (NB100-74340) was from Novus Biologicals, Littleton, CO, USA. Mouse monoclonal anti-LAMP1 (555798) was from BD Biosciences. Rabbit polyclonal
anti-TGN46 (AHP1586) and sheep polyclonal anti-TGN46 (AHP500) were from AbD Serotec, Oxford, UK. Rabbit polyclonal anti-RAB11A (TA324158) and

RAB11B (TA346586) were from Origene, Rockville, MD, USA. Mouse monoclonal anti-active- $\alpha 5$-integrin, SNAKA51, was previously described ${ }^{34}$. Rabbit polyclonal anti-Rab21 was kindly gifted from Johanna Ivaska, Turku Centre for

Biotechnology, University of Turku, Finland. Antigen, provider and dilution used

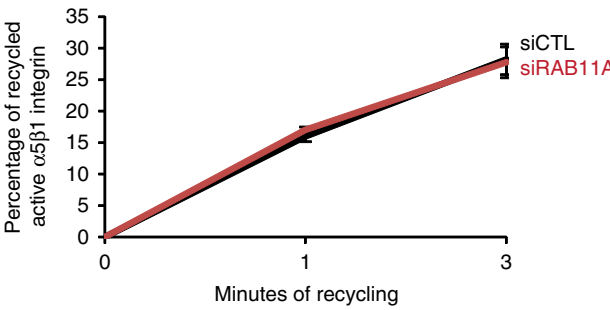

b
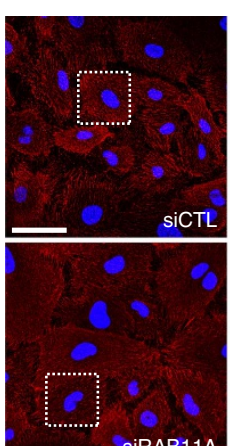

SIRAB11A

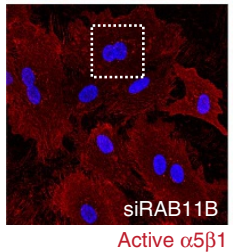

d

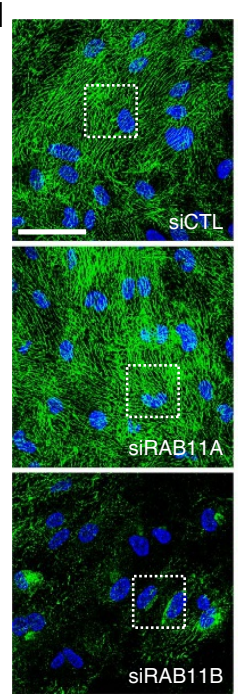

DAPI

ED-A FN

DAPI
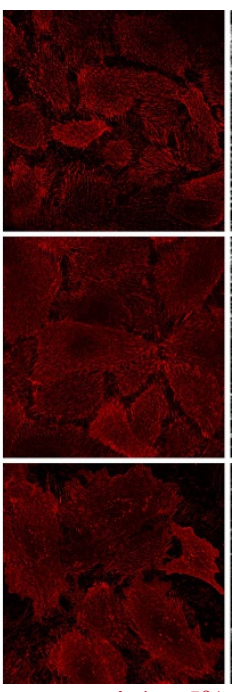

Active $\alpha 5 \beta 1$
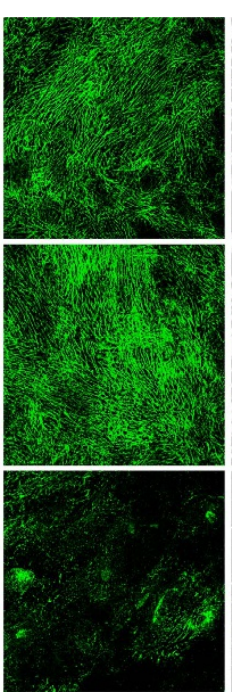

ED-A FN

e

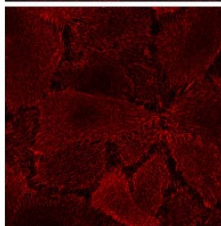

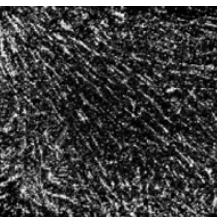
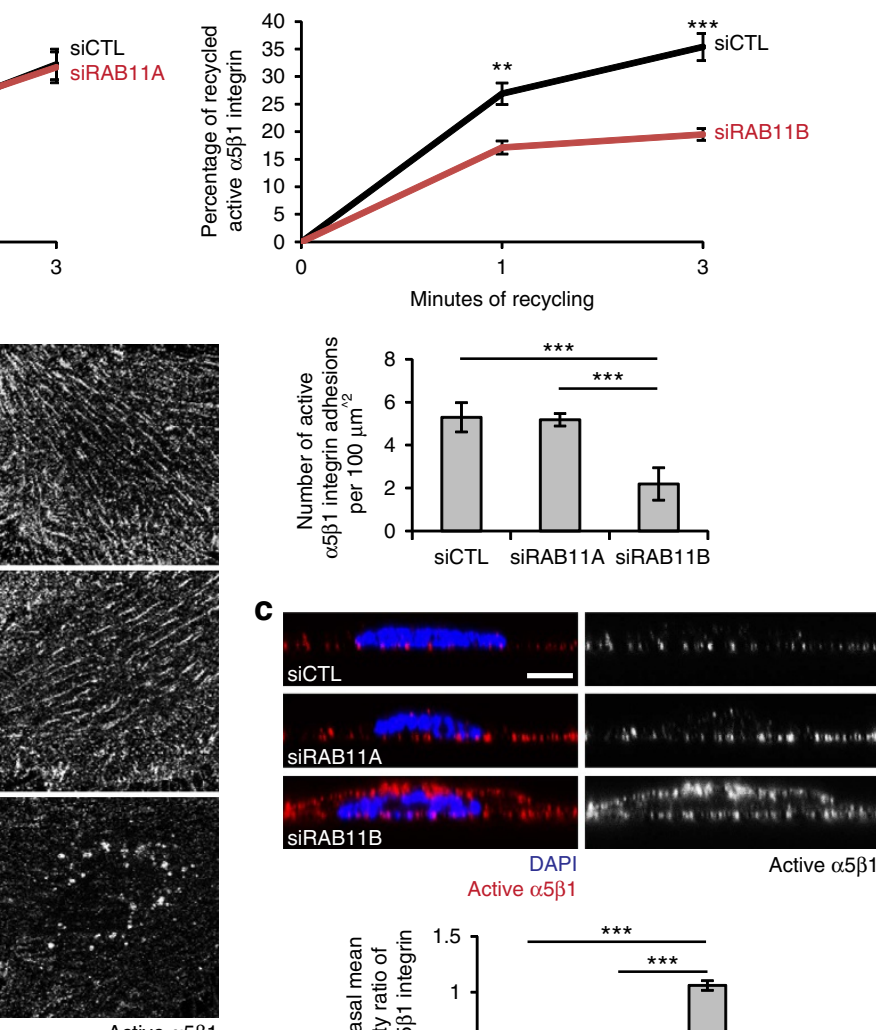

Active $\alpha 5 \beta 1$
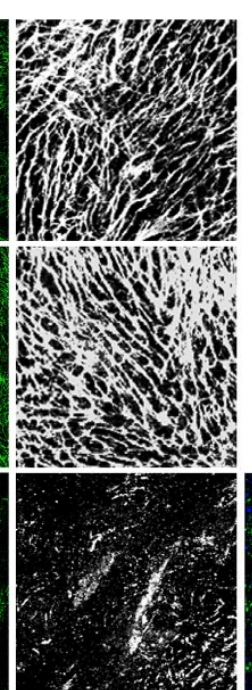

ED-A FN
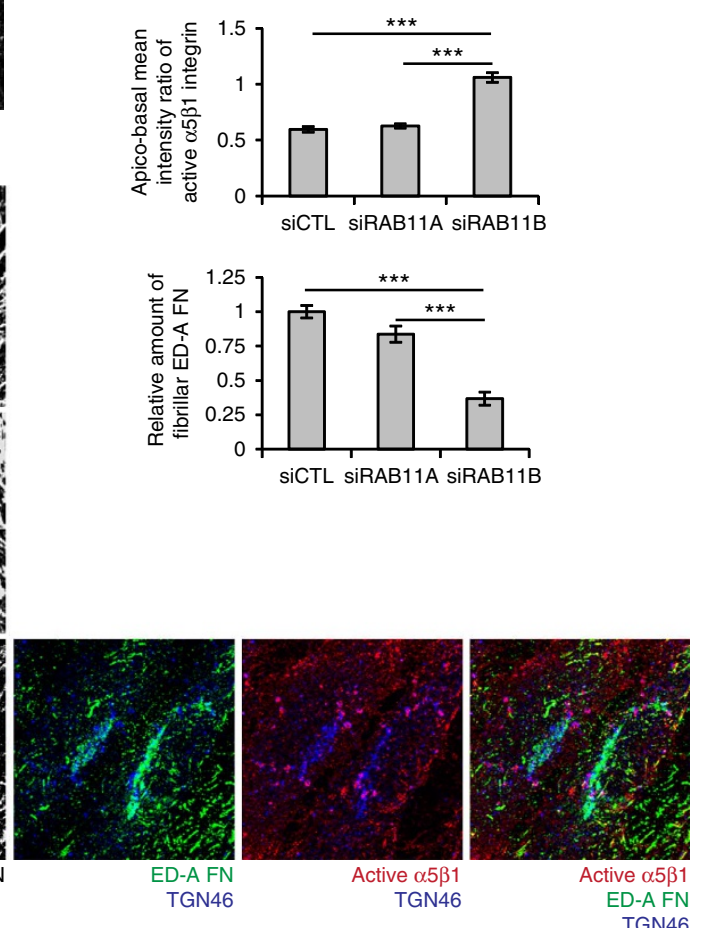

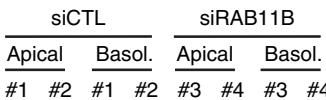
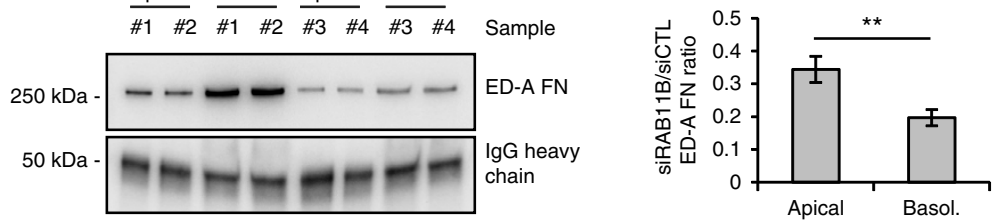
in different experimental settings for all the commercial and non-commercial primary antibodies employed in this study are summarized in Supplementary Table 3.

Goat anti-rabbit (sc-2054) and donkey anti-goat (sc-2056) HRP secondary antibodies were from Santa Cruz Biotechnology, while goat anti-mouse (115-035-003) HRP secondary antibody was from Jackson ImmunoResearch Laboratories, West Grove, PA, USA. Alexa Fluor 555 goat anti-mouse IgG2a (A21137), Alexa Fluor 488 goat anti-mouse IgG1 (A21121), Alexa Fluor 647 (A31571), 488 (A21202) and 555 (A31570) donkey anti-mouse IgG, Alexa Fluor 555 donkey anti-rabbit (A31572), Alexa Fluor 647 donkey anti-goat (A21447), Alexa Fluor 488 donkey anti-sheep (A11015) secondary antibodies, DAPI (D3571) and TO-PRO3 (T3605) were from Life technologies, Carlsbad, CA, USA.

Recombinant proteins. Human plasma FN (1918-FN-02M) was from R\&D Systems, Minneapolis, MN, USA. Rhodamine-labelled bovine FN (FNR01-A) was from Cytoskeleton, Denver, CO, USA. Cellular ED-A FN isolated from human foreskin fibroblasts, (F2518) was from Sigma Aldrich.

Cell culture. Primary arterial ECs were isolated from the umbilical cords as previously described ${ }^{14}$. Briefly, umbilical artery was cannulated with a blunt 17 -gauge needle and the needle was secured by clamping the cord over the needle. The artery was perfused with $50 \mathrm{ml}$ of PBS to wash out the blood. A total of $10 \mathrm{ml}$ of $0.2 \%$ collagenase A (Cat. No. 11088793001, Roche Diagnostics, Risch-Rotkreuz, Switzerland) in cell culture medium was then infused into the umbilical artery and incubated $30 \mathrm{~min}$ at room temperature. The collagenase solution containing the ECs was flushed from the cord by perfusion with $40 \mathrm{ml}$ of PBS, collected in a sterile $50 \mathrm{ml}$ centrifuge tube and centrifuged $5 \mathrm{~min}$ at $800 \mathrm{~g}$. Cells were resuspended in Endothelial Cell Growth Basal Medium (EBM-2) supplemented with EGM-2 BulletKit (Lonza Basel, Switzerland) (EGM-2) and plated in cell culture dishes adsorbed with $1 \%$ gelatin from porcine skin (G9136, Sigma Aldrich). Cells were tested for mycoplasma contamination by means of Venor GeM Mycoplasma Detection Kit (MP0025-1KT, Sigma Aldrich) and grown in EGM-2. The isolation of primary arterial ECs human umbilical cords was approved by the Office of the General Director and Ethics Committee of the Azienda Sanitaria Ospedaliera Ordine Mauriziano di Torino hospital (protocol approval no. 586, Oct 222012 and no. 26884, Aug 28 2014) and informed consent was obtained from each patient.

DNA constructs. Human PPFIA1 cDNA (transcript variant 1, NM_003626) from Origene Technologies Rockville, MD, USA was used as a template. PPFIA1r mutant construct was obtained by PCR, according to the Taq polymerase manufacturer's instructions, using Phusion Site-Directed Mutagenesis Kit

(Life technologies). Forward primers, containing two silent point mutation on each sequence targeted by siRNA, are:

Fw1_AAGAAAGGTTGCGACAAATGGAAGCAC

Fw2_GAACGACACTGGCGTATGGGGACATG;

Fw3_GATCGAGTGATACGGTGGATCCTGTC;

Fw4_AGGTGAGAGAACGATTGCGAGTAGCAC.

Afterwards, mutants were subcloned in pLVX-IRES-Puro lentiviral vector (Clontech, Mountain View, CA, USA), using standard PCR protocols, using Phusion High-Fidelity DNA Polymerase (Life technologies). Lentiviral particles were produced as described in the manufacturer's guidelines. pmCherry Vinculin was a gift from Kenneth Yamada, NIH, Bethesda, MD (Addgene plasmid \#50527). ST-GFP was generated by Dr Ian Trowbridge ${ }^{60}$ and kindly provided by Julia Von Blume, Max Planck Institute for Biochemistry, Martinsried, Germany.
Gene silencing. The day before oligofection, ECs were seeded in six-well dishes at a concentration of $10 \times 10^{4}$ cells per well. Oligofection of siRNA duplexes was performed according to the manufacturer's protocols. Briefly, human ECs were transfected twice (at 0 and $24 \mathrm{~h}$ ) with $200 \mathrm{pmol}$ of siCONTROL nontargeting siRNA (as control), siGENOME SMART pools siRNA oligonucleotides

(GE, Dharmacon, Lafayette, CO, USA), using Oligofectamine Transfection Reagent (Life technologies). $24 \mathrm{~h}$ after the second oligofection, ECs were lysed or tested in functional assays.

Immunoprecipitation and western blotting. ECs were lysed in a buffer containing $25 \mathrm{mM}$ Tris- $\mathrm{HCl}, \mathrm{pH} 7.6,100 \mathrm{mM} \mathrm{NaCl}, 0.15 \%$ Tween-20, 5\% glycerol, $0.5 \mathrm{mM}$ ethylene glycol tetraacetic acid (EGTA) and Protease Inhibitor Cocktail (Sigma Aldrich), $2 \mathrm{mM}$ phenylmethanesulfonylfluoride (PMSF), $2 \mathrm{mM} \mathrm{MgCl}$. Cell lysates were incubated for $20 \mathrm{~min}$ on wet ice, and then centrifuged at $15,000 \mathrm{~g}$, $20 \mathrm{~min}$, at $4^{\circ} \mathrm{C}$. The total protein amount was determined using the bicinchoninic acid (BCA) protein assay reagent (Life technologies). Equivalent amounts $(800 \mu \mathrm{g})$ of protein were immunoprecipitated for $1 \mathrm{~h}$ with the antibody of interest, and immune complexes were recovered on Protein G-Sepharose (GE Healthcare). Immunoprecipitates were washed twice with lysis buffer, twice with the same buffer without Tween-20 and then separated by SDS-PAGE. Proteins were then transferred to a nitrocellulose membrane (Biorad, Hercules, CA, USA), probed with antibodies of interest and detected by an enhanced chemiluminescence technique (PerkinElmer, Waltham, MA, USA).

Uncropped scans of all blots are provided as Supplementary Figs 11-14.

Pull-down assays. For bacterial expression, the intracellular domain of $\beta 1$ integrin ( $\beta 1$-cyto; residues from 752 to 799 of the full length mouse protein) or $\alpha 5$ integrin ( $\alpha 5$-cyto; residues from 1,026 to 1,054 of the full length mouse protein) were cloned into the pET15b vector (Clontech) in frame with the dimerization domains of Fos (residues from 161 to 200 of full length mouse protein) and Jun (residues from 277 to 318 of full length mouse protein) respectively. The $6 \times$ His tag sequence in pET15b vector (Clontech) was replaced with $3 \times$ Flag tag and Myc tag to generate Flag-Jun- $\alpha 5$ cyto, Flag-Fos- $\beta 1$ cyto and Myc-Jun- $\alpha 5$ cyto constructs.

Constructs were transformed into BL21 (DE3) Arctic Express Escherichia coli (Agilent Technologies, Santa Clara, CA, USA) and protein expression was induced with $0.8 \mathrm{mM}$ IPTG for $3 \mathrm{~h}$ at $30^{\circ} \mathrm{C}$. Afterwards, bacteria were pelleted by centrifugation $1485 \mathrm{~g}, 4^{\circ} \mathrm{C}$, resuspended in TBS buffer $(50 \mathrm{mM}$ Tris $\mathrm{pH} 7.4$, $150 \mathrm{mM} \mathrm{NaCl}$ ) containing Lysozyme $100 \mu \mathrm{g} \mathrm{ml}^{-1}$ and DNase $50 \mu \mathrm{g} \mathrm{ml}^{-1}$ and rotated for $2 \mathrm{~h}$ at $4{ }^{\circ} \mathrm{C}$. After the addition of Empigen (30\% solution) ( $1 \mathrm{ml} \mathrm{per}$ $10 \mathrm{ml}$ of lysate) the bacterial lysates were rotated for $1 \mathrm{~h}$ at $4{ }^{\circ} \mathrm{C}$ and centrifuged $1,485 \mathrm{~g}$ for $1 \mathrm{~h}$ at $4{ }^{\circ} \mathrm{C}$. Flag-Jun- $\alpha 5$, Flag-Fos- $\beta 1$ and a mixture of Flag-Jun- $\alpha 5$ and Myc-Fos- $\beta 1$ protein supernatants were incubated with ANTI-FLAG M2 Affinity Gel (Sigma, A2220) $2 \mathrm{~h}$ at $4{ }^{\circ} \mathrm{C}$ followed by extensive washing of the beads three times with TBS buffer. In parallel, mouse fibroblast cell lysate were lysed by for $10 \mathrm{~min}$ on ice with lysis buffer $(50 \mathrm{mM}$ Tris- $\mathrm{HCl}(\mathrm{pH} 7.5), 150 \mathrm{mM} \mathrm{NaCl}, 1 \%$ Triton X-100 and protease inhibitors (Roche, Basel, Switzerland), phosphatase inhibitor cocktail 2 and 3 (Sigma) and cleared by centrifugation. Overall, $1 \mathrm{mg}$ of cell lysate was added to the beads and incubated for $2 \mathrm{~h}$ at $4{ }^{\circ} \mathrm{C}$ with gentle agitation. After several washes with lysis buffer, proteins were subjected to SDSPAGE and western blotting with indicated antibodies.

Integrin recycling assay. Integrin recycling assays were performed as previously described by Roberts et al..$^{38}$ with minor modifications. Cells were transferred to ice, washed twice in cold phosphate-buffered saline (PBS) and surface-labelled at $4^{\circ} \mathrm{C}$ with $0.2 \mathrm{mg} \mathrm{ml}^{-1}$ sulfo-NHS-SS-biotin (Life technologies) in PBS for $30 \mathrm{~min}$

Figure 8 | RAB11B controls the recycling of endocytosed active $\alpha \mathbf{5 \beta 1}$ integrins and polarized ED-A FN secretion in ECs. (a) Time-course analysis of recycled active $\alpha 5 \beta 1$ integrin in siCTL ECs versus siRAB11A ECs or siRAB11B ECs. RAB11B, but not RAB11A, silencing significantly impairs active $\alpha 5 \beta 1$ integrin recycling. Data are mean \pm s.e.m., $n=3$ independent experiments (two technical replicates for each experiment). (b) Confocal microscopy analysis of anti-active $\alpha 5 \beta 1$ integrin mAb SNAKA51 localization (red) in living confluent ECs (20 min of incubation). SNAKA51 ${ }^{+}$active $\alpha 5 \beta 1$ integrin localizes in fibrillar adhesion in SiCTL and in siRAB11A, but not in siRAB11B ECs in which it accumulates in perinuclear punctae. The number of active $\alpha 5 \beta 1$ integrin-containing adhesions per $100 \mu \mathrm{m}^{2}$ of cell area was quantified in siCTL, siRAB11A and siRAB11B ECs. Data are mean values \pm s.e.m., $n=20$ cells per condition pooled from two independent experiments. (c) Confocal xz sectioning microscopy analysis of anti-active $\alpha 5 \beta 1$ integrin mAb SNAKA51 localization (red) in living confluent ECs (20 min of incubation). Quantitative analysis of apico-basal mean intensity ratio reveals that SNAKA51+ active $\alpha 5 \beta 1$ integrin localizes on the basolateral surface of siCTL and siRAB11A, but not of siRAB11B ECs, where it redistributes all around the cell surface. Data are mean values \pm s.e.m., $n=20$ cells per condition pooled from two independent experiments. (d) Confocal microscopy analysis of IST9 mAb ${ }^{+}$endogenous cellular ED-A FN (green) in confluent ECs. ED-A FN polymerizes into a fibrillar network in SiCTL and siRAB11A, but not in siRAB11B ECs, where it accumulates in a perinuclear compartment. Relative amount of fibrillary ED-A FN area was calculated in siCTL, siRAB11A and siRAB11B ECs. Data are mean values \pm s.e.m., $n=20$ cells per condition pooled from 2 independent experiments. (e) Western blot analysis of soluble ED-A FN released by confluent ECs seeded on Transwell inserts. An equal percentage of apical and basolateral volumes of medium were collected after $72 \mathrm{~h}$ of culture from different wells of siCTL or siRAB11B ECs. Equal amounts of rabbit lgG were exogenously added to samples (spike normalization) for loading control purposes. Quantification of the ratio between apical or basolateral amount of ED-A FN released by siCTL over siRAB11B ECs. RAB11B silencing much more severely impairs basolateral than apical ED-A FN secretion. Data are mean \pm s.e.m., $n=6$ wells per condition pooled from three independent experiments. Scale bar, $50 \mu m$ (d), $20 \mu \mathrm{m}(\mathbf{b}), 5 \mu \mathrm{m}(\mathbf{c}) .{ }^{\star \star} P<0.01 ;{ }^{\star \star \star} P<0.001$; Student's $t$-test. 
a

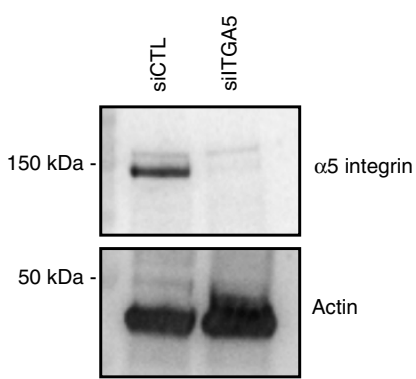

b
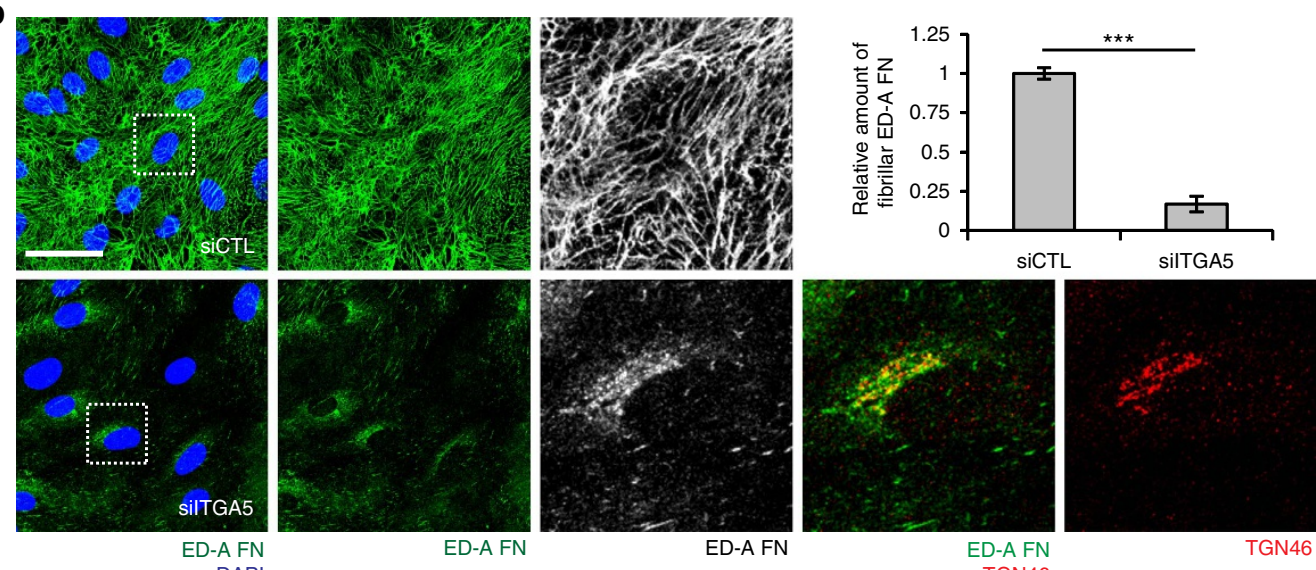

c

SiCTL
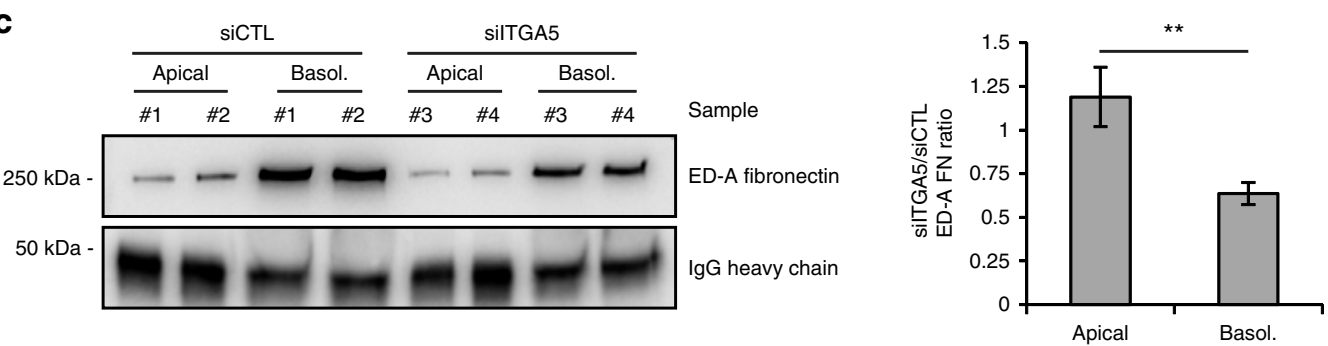

Figure 9 | $\mathbf{\alpha 5 \beta 1}$ regulates ED-A FN secretion and polymerization. (a) Western blot analysis of lysates of ECs control (siCTL) and $\alpha 5$ integrin subunit (silTGA5) silenced ECs. Cells were lysed 24 hours after the second siRNA oligofection and proteins were separated by SDS-PAGE and probed for $\alpha 5$ integrin subunit or actin (for control purposes). (b) Confocal microscopy analysis of IST9 mAb-labelled endogenous ED-A FN (green) in confluent ECs. ED-A FN polymerizes into a fibrillar network in siCTL, but not in silTGA5 ECs in which it accumulates in the TGN46+ (red) TGN cisternae. The relative amount of fibrillar ED-A FN area was calculated in siCTL and silTGA5 ECs. Data are mean \pm s.e.m., $n=20$ cells per condition pooled from two independent experiments. ${ }^{\star \star \star} P<0.001$; Student's $t$-test. (c) Western blot analysis of soluble ED-A FN released by confluent ECs seeded on Transwell inserts. An equal percentage of apical and basolateral volumes of medium were collected after $72 \mathrm{~h}$ of culture, from different wells of siCTL or silTGA5 ECs. Equal amounts of exogenous rabbit IgG were added to samples (spike normalization) for loading control purposes. Quantification of the ratio between apical or basolateral amount of ED-A FN released by siCTL over silTGA5 ECs. $\alpha 5$ integrin subunit silencing impairs basolateral, but not apical ED-A FN secretion. Data are mean \pm s.e.m., $n=8$ wells per condition pooled from four independent experiments. ${ }^{\star} P<0.01$; Student's $t$-test. Scale bar, $50 \mu \mathrm{m}(\mathbf{b})$.

Labelled cells were washed in cold PBS and transferred to prewarmed EGM-2 at $37^{\circ} \mathrm{C}$. After 5,10 or $15 \mathrm{~min}$, the medium was aspirated, and dishes were rapidly transferred to ice and washed twice with ice-cold PBS. Biotin was removed from proteins remaining at the cell surface by incubation with a solution containing $20 \mathrm{mM}$ sodium 2-mercaptoethanesulfonate (MesNa) in $50 \mathrm{mM}$ Tris- $\mathrm{HCl}(\mathrm{pH} \mathrm{8.6)}$, $100 \mathrm{mM} \mathrm{NaCl}$ for $1 \mathrm{~h}$ at $4{ }^{\circ} \mathrm{C}$. MesNa was quenched by the addition of $20 \mathrm{mM}$ iodoacetamide (IAA) for $10 \mathrm{~min}$, and after ECs were reicubated at $37^{\circ} \mathrm{C}$ for the indicated times. Afterwards, the medium was aspirated, and dishes were rapidly transferred to ice and washed twice with ice-cold PBS, biotin was removed from proteins recycled at the cell surface with MesNa and $\mathrm{MesNa}$ was quenched by IAA. ECs were lysed in $25 \mathrm{mM}$ Tris- $\mathrm{HCl}, \mathrm{pH} 7.4,100 \mathrm{mM} \mathrm{NaCl}, 2 \mathrm{mM} \mathrm{MgCl}, 1 \mathrm{mM}$ $\mathrm{Na}_{3} \mathrm{VO}_{4}, 0.5 \mathrm{mM}$ EGTA, $1 \%$ Triton X-100, 5\% glycerol, Protease Inhibitor Cocktail (Sigma Aldrich) and $1 \mathrm{mM}$ PMSF. Lysates were cleared by centrifugation at $12,000 \mathrm{~g}$ for $20 \mathrm{~min}$. Supernatants were corrected to equivalent protein concentrations and levels of biotinylated integrin were determined by capture ELISA assay.

Capture ELISA assay. Corning 96 Well Clear Polystyrene High Bind Stripwell Microplate (product \#2592, Corning, Corning, NY, USA) were coated overnight with $5 \mu \mathrm{g} \mathrm{ml}^{-1}$ appropriate anti-integrin antibodies in $0.05 \mathrm{M} \mathrm{Na}_{2} \mathrm{CO}_{3}(\mathrm{pH} 9.6)$ at $4{ }^{\circ} \mathrm{C}$ and were blocked in PBS containing $0.05 \%$ Tween-20 (PBS-T) with 5\% BSA for $1 \mathrm{~h}$ at RT. Integrins were captured by overnight incubation of $50 \mu \mathrm{l}$ cell lysate at $4{ }^{\circ} \mathrm{C}$. Unbound material was removed by extensive washing with PBS-T, and wells were incubated with streptavidin-conjugated horseradish peroxidase (GE Healthcare) in PBS-T containing $1 \%$ BSA for $1 \mathrm{~h}$ at $4{ }^{\circ} \mathrm{C}$. Following further washing, biotinylated integrins were detected by a chromogenic reaction with ortho-phenylenediamine.

Conventional and g-STED confocal scanning microscopy. Cells were plated on $0.17 \mathrm{~mm}$ glass coverslips (no. 1.5) coated with $1 \%$ gelatin (in PBS) or $3 \mu \mathrm{g} \mathrm{ml}^{-1} \mathrm{FN}$ and allowed to adhere overnight. Cells were washed in PBS, fixed in $4 \%$ paraformaldehyde (PFA), permeabilized in $0.1 \%$ Triton X-100 for 2 min on ice, incubated with different primary antibodies for $1 \mathrm{~h}$ at RT, and revealed by appropriate Alexa-Fluor-tagged secondary antibody (Life Technologies). Cells were analyzed by using a Leica TCS SP2 AOBS confocal laser-scanning microscope (Leica Microsystems, Wetzlar, Germany). A Leica TCS SP8 gated stimulated emission depletion (g-STED) $3 \times$ laser-scanning microscope was used to acquire super-resolved images (Leica Microsystems). A Leica STED HC PL APO $100 \times / 1.40$ objective was 
used. Fluorochromes and fluorescent proteins were excited at the optimal wavelength by means of $80 \mathrm{MHz}$ pulsed white light laser $(470-670 \mathrm{~nm})$, allowing time gating of fluorescence life times. For STED, the appropriate, 592 or $660 \mathrm{~nm}$, depletion laser was used. Fluorescence channels were scanned sequentially and emission was revealed by means of hybrid spectral detectors (HyD SP Leica Microsystems).
To quantify the localization of active $\alpha 5 \beta 1$ integrin in fibrillar adhesions, we counted the number of SNAKA mAb positive objects using Image $J$ shape descriptor AR (aspect ratio), selecting only the ones displaying a major_axis/ minor_axis. ratio $\geq 2$ per $100 \mu \mathrm{m}^{2}$ of cell area.

To quantify the apico-basal distribution of active $\alpha 5 \beta 1$ integrin, we measured mean fluorescence intensity for membrane length unit of SNAKA51 antibody for a
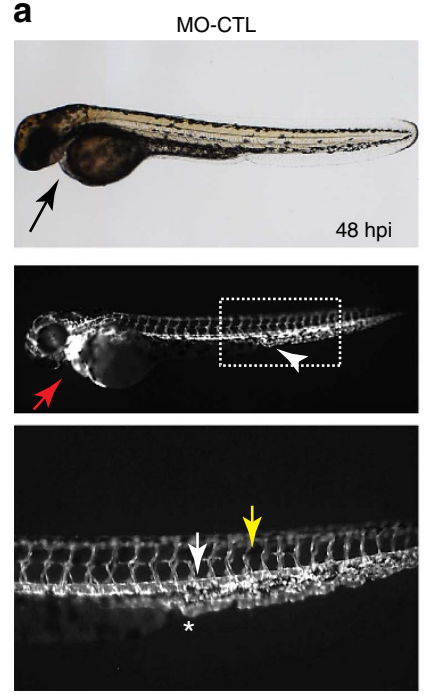

b

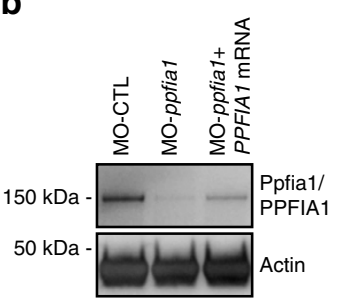

MO-ppfia1

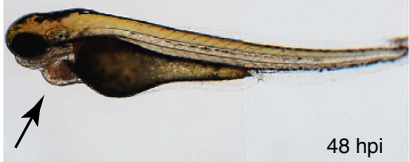

$48 \mathrm{hpi}$
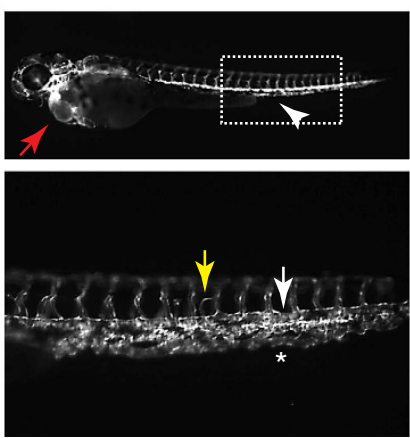

C

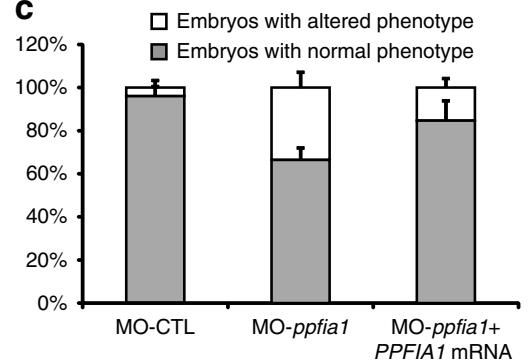

PPFIA1 mRNA d
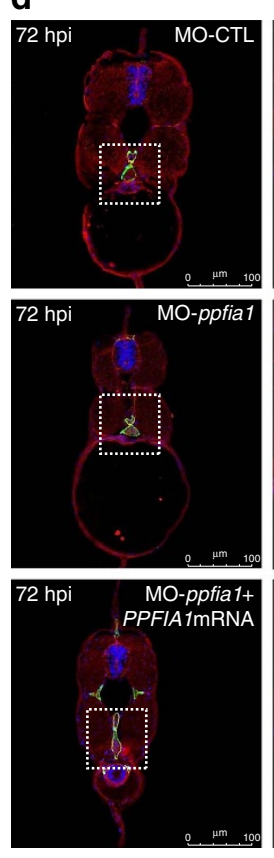

Fn1a $k d r l: E G F P$ DAPI
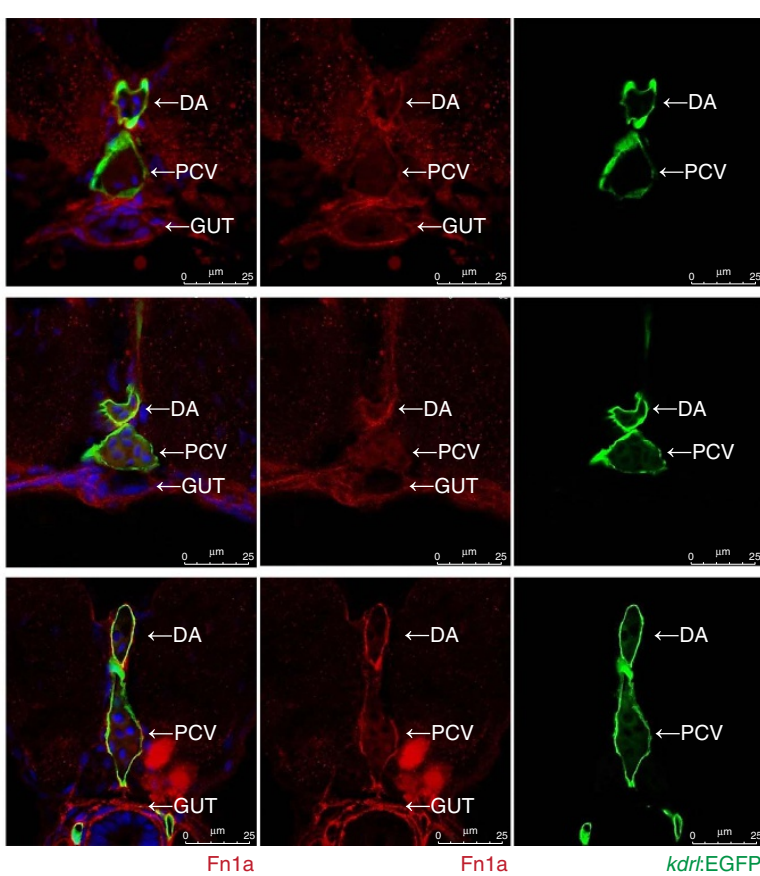
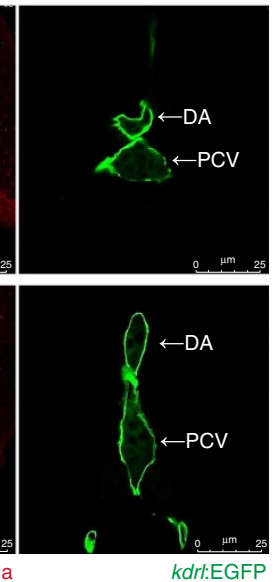

MO-ppfia1+ PPFIA1-mRNA
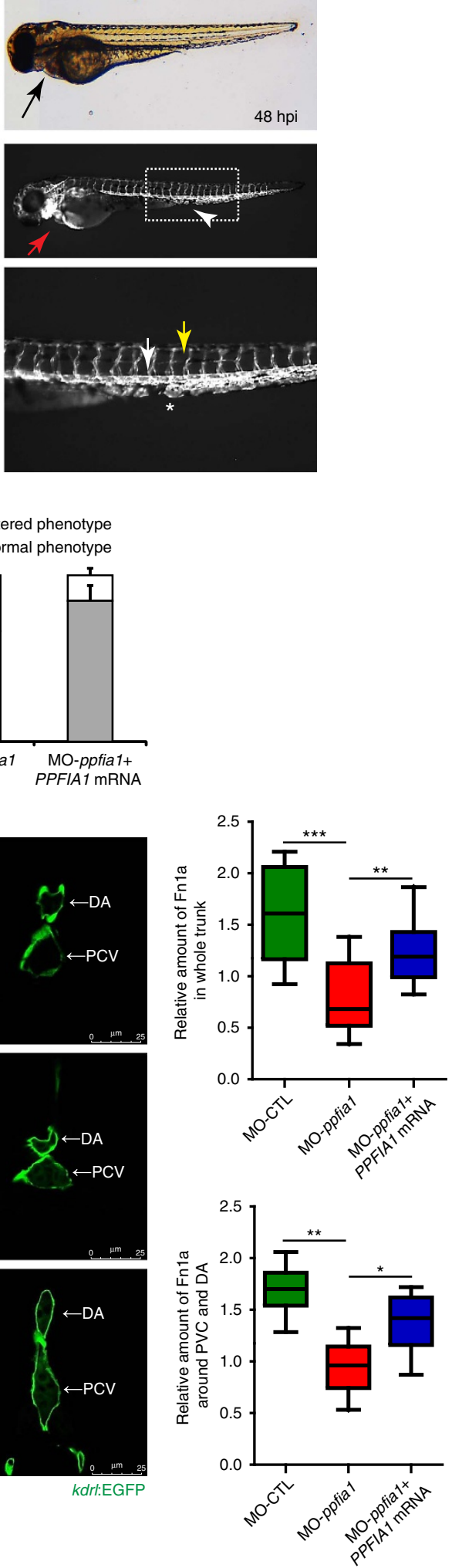
apical and basolateral membrane measuring total fluorescence intensity on the two membranes using Image J quantification tool and dividing it for relative membrane length. We reported in the histogram the ratio between apical and basolateral mean fluorescence intensity. Image acquisition was performed by adopting a laser power, gain and offset settings that allowed maintaining pixel intensities (grey scale) within the $0-255$ range and hence avoided saturation.

Internalization assays. Cells were plated on $0.17 \mathrm{~mm}$ glass coverslips (no. 1.5) coated with $1 \%$ gelatin (in PBS) or $3 \mu \mathrm{g} \mathrm{ml}^{-1} \mathrm{FN}$ and allowed to adhere overnight. Cells were incubated either with anti-active $\alpha 5$ integrin subunit SNAKA51 mAb or anti-ED-A FN IST9 $\mathrm{mAb}$ for $20 \mathrm{~min}$ at $37^{\circ} \mathrm{C}$, then washed with PBS and incubated $0.5 \mathrm{M} \mathrm{NaCl}, 0.5 \%$ acetic acid, $2.6 \mathrm{pH}$ for $6 \mathrm{~min}$ at $4{ }^{\circ} \mathrm{C}$ to remove the antibodies bound on the cell surface. Cells were then washed thrice with PBS, fixed with $4 \%$ PFA and processed for immunofluorescence. The amount of internalized active $\alpha 5 \beta 1$ integrin or ED-A FN was quantified as mean fluorescence intensity of SNAKA51 or IST9 antibodies in EEA positive or TGN46 positive structures by means of Image J software.

FN fibrillogenesis. Small interfering RNA silencing was performed, and, after the second oligofection, cells were seeded onto gelatin coated $0.17 \mathrm{~mm}$ glass coverslips (no. 1.5) in 24-well dishes at a concentration of $15 \times 10^{4}$ cells per well or in 6-well dishes at a concentration of $4 \times 10^{5}$ cells per well and left to adhere for $16 \mathrm{~h}$ in EGM-2 medium containing FN-depleted serum. Cells on coverslips were incubated with SNAKA51 mAb for $20 \mathrm{~min}$ at $37^{\circ} \mathrm{C}$, then washed with PBS, fixed with $4 \%$ PFA and processed for immunofluorescence. The amount of ED-A FN fibrils was quantified as the fraction of cell area stained by IST $9 \mathrm{mAb}$ by means of Image J software.

Cells plated in 6-well dishes were either processed for sodium deoxycholate (DOC) solubility assay or lysed in hot SDS lysis buffer to analyze protein expression by western blot. DOC solubility assay was performed as previously described $^{61}$ with minor modifications. Briefly, ECs were put on ice and lysed with cold DOC lysis buffer (2\% DOC; $20 \mathrm{mM}$ Tris-HCl, $\mathrm{pH}$ 8.8; $2 \mathrm{mM}$ PMSF; $2 \mathrm{mM}$ EDTA; $2 \mathrm{mM}$ iodoacetic acid; $2 \mathrm{mM} \mathrm{N}$-ethylmaleimide). EC lysates were collected with a $26 \mathrm{G}$ needled syringe and immediately centrifuged at $18,800 \mathrm{~g}$, for $15 \mathrm{~min}$ at $4{ }^{\circ} \mathrm{C}$. The supernatant was removed; the pellet was washed once with DOC buffer and solubilized in $25 \mu \mathrm{l}$ of SDS buffer (1\% SDS; $20 \mathrm{mM}$ Tris- $\mathrm{HCl}, \mathrm{pH} 8.8 ; 2 \mathrm{mM}$ PMSF; $2 \mathrm{mM}$ EDTA; $2 \mathrm{mM}$ iodoacetic acid; $2 \mathrm{mM}$ N-ethylmaleimide). Protein concentration in DOC-soluble fractions was estimated using BCA protein assay, and used to normalize protein concentration in the corresponding DOC-insoluble fraction. DOC-insoluble samples were resolved by SDS-PAGE using 7.5\% polyacrylamide gel, transferred to nitrocellulose and immunoblotted with IST9 mAb.

Apico-basal FN secretion assay. ECs were seeded onto gelatin coated polycarbonate membrane Transwell inserts, pore $0.4 \mu \mathrm{m}$ (3413, Corning, Lowell, MA, USA), at a $15 \times 10^{4}$ cells per well density and left to adhere for $24 \mathrm{~h}$ in EGM-2 medium. Next, transendothelial Resistance (TER) was measured with Millicell ERS-2 Volt-Ohm Meter (Millipore) to verify endothelial monolayer integrity and the medium was replaced with EGM-2 containing FN-depleted serum $(200 \mu \mathrm{l}$ in the upper chamber and $600 \mu \mathrm{l}$ in the lower chamber). After $72 \mathrm{~h}$, the medium was collected and cleared by centrifugation at $15,000 \mathrm{~g}, 20 \mathrm{~min}$, at $4^{\circ} \mathrm{C}$. Spike normalization was performed adding $5 \mu \mathrm{g}$ of rabbit anti-mouse IgG in the samples collected from upper and lower chambers and visualized in western blot for loading control purpose. Equal percentage of apical and basolateral volume of medium were resolved by SDS-PAGE using 7.5\% polyacrylamide gel, transferred to nitrocellulose, and immunoblotted with IST9 mAb or anti-rabbit IgG-HRP.

Time-lapse TIRF microscopy. TIRF microscopy on living ECs was performed using a Leica AM TIRF MC system mounted on a Leica AF 6000LX workstation. Cells were plated onto glass-bottom dishes (WillCo-dish; WillCo Wells B.V., Amsterdam, The Netherlands) and placed onto a sample stage within an incubator chamber set to $37^{\circ} \mathrm{C}$, in an atmosphere of $5 \% \mathrm{CO}_{2}, 20 \%$ humidity. A Leica HC PI APO $63 \times / 1.47 \mathrm{NA}$ oil-immersion objective was used, and laser penetration depth was set at $90 \mathrm{~nm}$. Excitation and analysis of fluorescent proteins were performed with a $488 \mathrm{~nm}$ (for GFP) and $532 \mathrm{~nm}$ (for Cherry) laser. Imaging was recorded on a Hamamatsu EM-CCD camera (C9100-02, Hamamatsu, SZK, Japan).

In vitro angiogenesis assay. Overall, 17,000 ECs were resuspended in $350 \mu \mathrm{l}$ of $10 \%$ FBS EBM-2, plated in each well of a 48 -well plate previously coated with $150 \mu \mathrm{l}$ per well of $8 \mathrm{mg}$ per $\mathrm{ml}$ growth factor-reduced Matrigel (BD Biosciences, Franklin Lakes, NJ, USA) and incubated at $37^{\circ} \mathrm{C}, 5 \% \mathrm{CO}_{2}$ in a humidified atmosphere for $8 \mathrm{~h}$ to allow vascular network formation. Samples were observed by a Leica DMI 3000 B inverted microscope (Leica Microsystems). Images were acquired with a CoolSNAP HQ camera (Photometrics, Tucson, AZ, USA) controlled by Image Pro Plus software (Media Cybernetics, Rockville, MD, USA).

Generation and analyses of zebrafish morphants. The adults and embryo fishes were raised and maintained under standard laboratory conditions. Experimental procedures related to fish manipulation followed previously reported recommendations $s^{62}$ and conformed to the Italian regulations for protecting animals used in research, including DL 116/92. The Ethics committee of the University of Torino approved this study. Larvae were anesthetized and, then, sacrifice by ice chilling.

The transgenic zebrafish line $\left(T g(K d r l: G F P)^{s 843}\right)$ carrying endothelial-specific expression of the EGFP, was used in the described experiments. Morpholinos were injected in the zebrafish eggs at single-cell stage using a $\mathrm{CO}_{2}$-based Picospritzer-III device and the phenotype assessed after 48 hpi. Knockdown of PPFIAl was achieved through the microinjection of the following morpholino: $5^{\prime}$-TGGTGGG CATCACCTCGCACATCAT $-3^{\prime}$. The following morpholino was used as PPFIA1 5- base mismatch control: $5^{\prime}$-TCGTGGCCATCAACTCGAACATAAT- $3^{\prime}$. Morpholinos were synthesized by Genetools and resuspended in nuclease-free water. The mixture of injection was prepared diluting each morpholino in fish water and phenol red 5\% to a final concentration of $83 \mu \mathrm{M}$. The volume of injection was set to $4 \mathrm{nl}$. The coding DNA sequence of PPFIA1 was PCR amplified and cloned into pCS2 ${ }^{+}$plasmid between ClaI and XhoI sites. The corresponding mRNA was prepared using an SP6 MessageMachine kit (Ambion) according to the manufacturer's indications and its size verified by gel electrophoresis. The in vitro synthesized capped mRNA was resuspended in nuclease-free water to a final concentration of $100 \mathrm{ngl}^{-1}$. For the rescue experiments, the mixture of injection was prepared diluting the PPFIA1 morpholino and PPFIA1 mRNA in fish water and phenol red $5 \%$ respectively to a final concentration of $83 \mu \mathrm{M}$ and $10 \mathrm{ng} \mu \mathrm{l}^{-1}$ The volume of injection was set to $4 \mathrm{nl}$.

For the Western blot analysis 50 embryos for each experimental group were placed in a pre-chilled $3.5 \mathrm{~cm}$ petri dish containing ice-cold PBS with the addition of proteases and phosphatases inhibitors (Roche). The yolk sac was removed passing each embryo several times through a $200 \mu \mathrm{l}$ tip. Deyolked embryos were subsequently transferred into new Eppendorf tubes and lysed in 2\% SDS lysis buffer at $90^{\circ} \mathrm{C}$ for $10 \mathrm{~min}$. DNA was removed by sonication of the lysates with 3 pulses of $30 \mathrm{sec}$ each. Lysates were then centrifuged for $15 \mathrm{~min}$ at $14,000 \mathrm{~g}$ and

Figure 10 | PPFIA1 silencing affects vascular morphogenesis in developing zebrafish embryo. (a) Lateral view at conventional light and confocal fluorescence microscopy of $\mathrm{Tg}(\mathrm{kdrl}: \mathrm{EGFP})$ zebrafish embryos carrying EC-specific EGFP expression and derived from fertilized zebrafish eggs that were injected with $83 \mu \mathrm{M}$ of a ppfia1 translation blocking morpholino (MO-ppfia1), 5-base mismatch (MO-CTL), or ppfia1 translation blocking morpholino and 40 pg PPFIA1 mRNA at the single-cell stage. Starting from 48 hpi, 35\% of the ppfia1 morphants displayed: (i) an enlarged heart chamber phenotype (red arrows) due to atrium dilation associated with pericardial oedema, blood stasis and reduced blood flow despite the presence of cardiac activity; (ii) malformations in the intersegmental vessels (Se, yellow arrow); (iii) an irregular shape (margin) in the DA and the PCV (white arrows). Human PPFIA1 mRNA co-injection successfully rescued the cardiovascular defects in the vast majority ( $\sim 84 \%$ ) of ppfial morphants that appeared morphologically normal (b). Western blot analysis of MO-ppfia1, MO-CTL and MO-ppfia1 + PPFIA1 mRNA morphants, at 48 hpi, revealed that the band corresponding to zebrafish ppfia1 present in MO-CTL, completely disappear in MO-ppfia1 and it is partially restored in MO-ppfia1 + PPFIA1 mRNA morphants. Actin was used as loading control. (c) Relative percentage of the embryos having normal or altered phenotype in two independent experiments is reported in the graph and both absolute numbers with relative percentage of the embryos for each experimental group are reported in Supplementary Tables 1 and 2. (d) Confocal fluorescence microscopy analysis of MO-CTL, MO-ppfia1 and MO-ppfia1 + PPFIA1 mRNA Tg(Kdrl:EGFP) zebrafish embryos trunk cross-sections at 72 hpi stained with DAPI and Fn1a. Right panels are magnifications of boxed areas of the left panels. While MO-CTL morphants show normal Fn1a expression around the PCV, DA, gut and in the whole trunk cross-section (upper panels); MO-ppfia1 morphants display a decreased Fn1a expression in the overall trunk region (middle panels). Co-injection of human PPFIA1 mRNA in MO-ppfia1 morphants rescued Fn1a deposition defects albeit not completely in the overall trunk cross-section. Relative amount of Fn1a expression in MO-CTL, MO-ppfia1 and MO-ppfia1 + PPFIA1 mRNA Tg(Kdrl:EGFP) zebrafish whole trunk cross-sections or around PVC and DA, at 72 hpi. Data are mean values \pm s.d. from 4 (MO-CTL) or 9 (MO-ppfia1 and MO-ppfia1+ PPFIA1 mRNA) embryos from two independent experiments, and 5th/95th percentile. ${ }^{\star} P<0.05$; ${ }^{\star \star} P<0.01$; ${ }^{\star \star \star} P<0.001$; ANOVA followed by the Tukey range test. Scale bar, $100 \mu \mathrm{m}$ (d, left panels), $25 \mu \mathrm{m}$ (d, right panels). 
protein concentration was determined with a detergent-compatible Lowry assay (DC Protein Assay, BioRad). Equal amounts of protein were resolved by SDS-PAGE using $7.5 \%$ polyacrylamide gel, transferred to nitrocellulose, and immunoblotted with the indicated antibodies.

For the immunofluorescence detection of Fnla, $\mathrm{Tg}(\mathrm{kdrl}: \mathrm{EGFP})$ zebrafish embryos were collected at $72 \mathrm{hpi}$, fixed overnight in $4 \%$ PFA at $4{ }^{\circ} \mathrm{C}$, washed twice in PBS-Tween $0.1 \%$, embedded in $4 \%$ low-melting agarose and therefore sectioned with a Leica vibratome to produce transversal cross-sections of $250 \mu \mathrm{m}$. After permeabilization with PBS-Tween $0.1 \%$, the sections were washed three times and incubated overnight at $4{ }^{\circ} \mathrm{C}$ with a rabbit primary antibody (Sigma Aldrich F3648) directed against Fn1a (1:200 dilution in PBS-Tween 0.1\%). The sections were washed three times in PBS-Tween $0.1 \%$ and therefore incubated $\mathrm{O} / \mathrm{N}$ at $4{ }^{\circ} \mathrm{C}$ with a secondary anti-rabbit antibody (Life Technologies) conjugated with Alexa Fluor568 (1:1,000 dilution in PBS-Tween $0.1 \%)$ and with $0.1 \%$ DAPI to counterstain the nuclei. After extensive washing in PBS-Tween $0.1 \%$, the sections were washed once in $\mathrm{dH}_{2} \mathrm{O}$ and mounted on glass slides with Vectashield (Vector Labs).

Zebrafish sections were imaged with a Leica TCS SP5 X confocal microscope equipped with a tandem scanning system with an oil-immersion objective HCX PL FLUOTAR $40 \times(\mathrm{NA} 1.25)$ and Leica LAS AF software. Images were acquired after sequential excitation at $405 \mathrm{~nm}$ for DAPI, $488 \mathrm{~nm}$ for GFP and $543 \mathrm{~nm}$ for Alexa Fluor-568 followed by emission detection at the appropriate wavelength using a frame resolution of $1,024 \times 1,024$ pixels, a pinhole opening of 1 Airy and a scanning frequency of $200 \mathrm{~Hz}$. For each section, z-stacks were acquired with $2 \mu \mathrm{m}$ distance. The $\mathrm{z}$-stacks were processed and analyzed by ImageJ. Background subtraction was performed using the rolling ball procedure set to 50 pixels and the pictures converted to 32-bit format. The integrated intensity for the three separated channels was calculated in both the total cross section and in the perivascular region surrounding the PCV and the DA vessels. The normalized FN values were calculated dividing the integrated intensity of the red channel (FN-1) for the integrated intensity of the blue channel (DAPI). The normalized ratio was plotted in Graphpad as box-wiskers plot.

Statistical analysis. For statistical evaluation, results were analyzed by a two-tailed heteroscedastic Student's $t$-test.

For the zebrafish embryo, experimental data were visualized by box-whisker plot showing the lower quartile (bottom box), the upper quartile (top box), median (band near the middle of the box), the fifth percentile (lower end of whisker) and the ninety-fifth percentile (upper end of whisker). For multiple comparisons, the data were analyzed with a one-way analysis of variance (ANOVA) followed by the Tukey range test. Stars indicate the following $P$-values: ${ }^{*} P<0.05 ;{ }^{* *} P<0.01$; ${ }^{* * *} P<0.001$. Neither randomization nor blinding was applied for zebrafish embryo analyses.

Data availability. The authors declare that all data supporting the findings of this study are available within the article and its Supplementary Information files or are available from the corresponding author upon request.

\section{References}

1. Astrof, S. \& Hynes, R. O. Fibronectins in vascular morphogenesis. Angiogenesis 12, 165-175 (2009)

2. Weber, G. F., Bjerke, M. A. \& DeSimone, D. W. Integrins and cadherins join forces to form adhesive networks. J. Cell Sci. 124, 1183-1193 (2011).

3. George, E. L., Baldwin, H. S. \& Hynes, R. O. Fibronectins are essential for heart and blood vessel morphogenesis but are dispensable for initial specification of precursor cells. Blood 90, 3073-3081 (1997).

4. Zovein, A. C. et al. Betal integrin establishes endothelial cell polarity and arteriolar lumen formation via a Par3-dependent mechanism. Dev. Cell 18, 39-51 (2010).

5. Iruela-Arispe, M. L. \& Davis, G. E. Cellular and molecular mechanisms of vascular lumen formation. Dev. Cell 16, 222-231 (2009).

6. Bryant, D. M. \& Mostov, K. E. From cells to organs: building polarized tissue. Nat. Rev. Mol. Cell Biol. 9, 887-901 (2008).

7. Zhou, X. et al. Fibronectin fibrillogenesis regulates three-dimensional neovessel formation. Genes Dev. 22, 1231-1243 (2008).

8. Schwarzbauer, J. E. \& DeSimone, D. W. Fibronectins, their fibrillogenesis, and in vivo functions. Cold Spring Harb. Perspect. Biol. 3, a005041 (2011).

9. Shi, F. \& Sottile, J. MT1-MMP regulates the turnover and endocytosis of extracellular matrix fibronectin. J. Cell Sci. 124, 4039-4050 (2011).

10. Shi, F. \& Sottile, J. Caveolin-1-dependent $\{$ beta $\} 1$ integrin endocytosis is a critical regulator of fibronectin turnover. J. Cell Sci. 121, 2360-2371 (2008).

11. Valdembri, D. \& Serini, G. Regulation of adhesion site dynamics by integrin traffic. Curr. Opin. Cell Biol. 24, 582-591 (2012).

12. Jacquemet, G., Humphries, M. J. \& Caswell, P. T. Role of adhesion receptor trafficking in 3D cell migration. Curr. Opin. Cell Biol. 25, 627-632 (2013).

13. De Franceschi, N., Hamidi, H., Alanko, J., Sahgal, P. \& Ivaska, J. Integrin traffic-the update. J. Cell Sci. 128, 839-852 (2015).
14. Valdembri, D. et al. Neuropilin-1/GIPC1 Signaling regulates $\alpha 5 \beta 1$ integrin traffic and function in endothelial cells. PLoS Biol. 7, e1000025 (2009).

15. Rainero, E. et al. Ligand-occupied integrin internalization links nutrient signaling to invasive migration. Cell Rep. 10, 398-413 (2015).

16. Dozynkiewicz, M. A. et al. Rab25 and CLIC3 collaborate to promote integrin recycling from late endosomes/lysosomes and drive cancer progression. Dev. Cell 22, 131-145 (2012).

17. Kowalczyk, A. P., Tulloh, R. H. \& McKeown-Longo, P. J. Polarized fibronectin secretion and localized matrix assembly sites correlate with subendothelial matrix formation. Blood 75, 2335-2342 (1990).

18. Kano, Y., Katoh, K., Masuda, M. \& Fujiwara, K. Macromolecular composition of stress fiber-plasma membrane attachment sites in endothelial cells in situ. Circ. Res. 79, 1000-1006 (1996).

19. Baldus, S. et al. Endothelial transcytosis of myeloperoxidase confers specificity to vascular ECM proteins as targets of tyrosine nitration. J. Clin. Invest. 108, 1759-1770 (2001).

20. Rodriguez-Boulan, E., Kreitzer, G. \& Müsch, A. Organization of vesicular trafficking in epithelia. Nat. Rev. Mol. Cell Biol. 6, 233-247 (2005).

21. De Matteis, M. A. \& Luini, A. Exiting the Golgi complex. Nat. Rev. Mol. Cell Biol. 9, 273-284 (2008).

22. Rodriguez-Boulan, E. \& Macara, I. G. Organization and execution of the epithelial polarity programme. Nat. Rev. Mol. Cell Biol. 15, 225-242 (2014).

23. Tiwari, A. et al. Endothelial cell migration on fibronectin is regulated by syntaxin 6-mediated alpha5betal integrin recycling. J. Biol. Chem. 286, 36749-36761 (2011).

24. Riggs, K. A. et al. Regulation of integrin endocytic recycling and chemotactic cell migration by syntaxin 6 and VAMP3 interaction. J. Cell Sci. 125, 3827-3839 (2012).

25. Reverter, M. et al. Cholesterol regulates Syntaxin 6 trafficking at trans-Golgi network endosomal boundaries. Cell Rep. 7, 883-897 (2014).

26. Shafaq-Zadah, M. et al. Persistent cell migration and adhesion rely on retrograde transport of $\beta 1$ integrin. Nat. Cell Biol. 18, 54-64 (2016).

27. Serra-Pages, C., Medley, Q. G., Tang, M., Hart, A. \& Streuli, M. Liprins, a family of LAR transmembrane protein-tyrosine phosphatase-interacting proteins. J. Biol. Chem. 273, 15611-15620 (1998).

28. Serra-Pages, C. et al. The LAR transmembrane protein tyrosine phosphatase and a coiled-coil LAR-interacting protein co-localize at focal adhesions. EMBO J. 14, 2827-2838 (1995).

29. Südhof, T. C. The presynaptic active zone. Neuron 75, 11-25 (2012).

30. Astigarraga, S., Hofmeyer, K., Farajian, R. \& Treisman, J. E. Three Drosophila liprins interact to control synapse formation. J. Neurosci. 30, 15358-15368 (2010).

31. Ziv, N. E. \& Garner, C. C. Cellular and molecular mechanisms of presynaptic assembly. Nat. Rev. Neurosci. 5, 385-399 (2004).

32. Haucke, V., Neher, E. \& Sigrist, S. J. Protein scaffolds in the coupling of synaptic exocytosis and endocytosis. Nat. Rev. Neurosci. 12, 127-138 (2011).

33. van der Vaart, B. et al. CFEOM1-associated kinesin KIF21A is a cortical microtubule growth inhibitor. Dev. Cell 27, 145-160 (2013).

34. Clark, K. et al. A specific alpha5beta1-integrin conformation promotes directional integrin translocation and fibronectin matrix formation. J. Cell Sci. 118, 291-300 (2005)

35. Su, Y. et al. Relating conformation to function in integrin $\alpha 5 \beta 1$. Proc. Natl Acad. Sci. USA 113, E3872-E3881 (2016).

36. Byron, A. et al. Anti-integrin monoclonal antibodies. J. Cell Sci. 122, 4009-4011 (2009).

37. Montanez, E. et al. Kindlin-2 controls bidirectional signaling of integrins. Genes Dev. 22, 1325-1330 (2008)

38. Roberts, M., Barry, S., Woods, A., van der Sluijs, P. \& Norman, J. PDGF-regulated rab4-dependent recycling of alphavbeta3 integrin from early endosomes is necessary for cell adhesion and spreading. Curr. Biol. 11, 1392-1402 (2001).

39. Arjonen, A., Alanko, J., Veltel, S. \& Ivaska, J. Distinct recycling of active and inactive $\beta 1$ integrins. Traffic 13, 610-625 (2012).

40. Wood, S. A., Park, J. E. \& Brown, W. J. Brefeldin A causes a microtubulemediated fusion of the trans-Golgi network and early endosomes. Cell 67, 591-600 (1991).

41. Waguri, S. et al. Visualization of TGN to endosome trafficking through fluorescently labeled MPR and AP-1 in living cells. Mol. Biol. Cell 14, 142-155 (2003).

42. Dugina, V., Fontao, L., Chaponnier, C., Vasiliev, J. \& Gabbiani, G. Focal adhesion features during myofibroblastic differentiation are controlled by intracellular and extracellular factors. J. Cell Sci. 114, 3285-3296 (2001).

43. Tzima, E., del Pozo, M. A., Shattil, S. J., Chien, S. \& Schwartz, M. A. Activation of integrins in endothelial cells by fluid shear stress mediates Rho-dependent cytoskeletal alignment. EMBO J. 20, 4639-4647 (2001). 
44. Pellinen, T. et al. Small GTPase Rab21 regulates cell adhesion and controls endosomal traffic of beta1-integrins. J. Cell Biol. 173, 767-780 (2006).

45. Sandri, C. et al. The R-Ras/RIN2/Rab5 complex controls endothelial cell adhesion and morphogenesis via active integrin endocytosis and Rac signaling. Cell Res. 22, 1479-1501 (2012).

46. Simpson, J. C. et al. A role for the small GTPase Rab21 in the early endocytic pathway. J. Cell Sci. 117, 6297-6311 (2004).

47. Gravotta, D. et al. The clathrin adaptor AP-1A mediates basolateral polarity. Dev. Cell 22, 811-823 (2012).

48. Welz, T., Wellbourne-Wood, J. \& Kerkhoff, E. Orchestration of cell surface proteins by Rab11. Trends Cell Biol. 24, 407-415 (2014).

49. Lapierre, L. A. et al. Rab11b resides in a vesicular compartment distinct from Rab11a in parietal cells and other epithelial cells. Exp. Cell Res. 290, 322-331 (2003).

50. Thuenauer, R. et al. Four-dimensional live imaging of apical biosynthetic trafficking reveals a post-Golgi sorting role of apical endosomal intermediates. Proc. Natl Acad. Sci. USA 111, 4127-4132 (2014).

51. Blum, M., De Robertis, E. M., Wallingford, J. B. \& Niehrs, C. Morpholinos: antisense and sensibility. Dev. Cell 35, 145-149 (2015).

52. Hoege, C. \& Hyman, A. A. Principles of PAR polarity in Caenorhabditis elegans embryos. Nat. Rev. Mol. Cell Biol. 14, 315-322 (2013).

53. Tam, P. P. \& Loebel, D. A. Gene function in mouse embryogenesis: get set for gastrulation. Nat. Rev. Genet. 8, 368-381 (2007).

54. Hynes, R. O. Integrins: bidirectional, allosteric signaling machines. Cell 110, 673-687 (2002).

55. Lee, J. L. \& Streuli, C. H. Integrins and epithelial cell polarity. J. Cell Sci. 127, 3217-3225 (2014).

56. Palamidessi, A. et al. The GTPase-activating protein RN-tre controls focal adhesion turnover and cell migration. Curr. Biol. 23, 2355-2364 (2013).

57. Parsons, J. T., Horwitz, A. R. \& Schwartz, M. A. Cell adhesion: integrating cytoskeletal dynamics and cellular tension. Nat. Rev. Mol. Cell Biol. 11, 633-643 (2010).

58. Trinh, L. A. \& Stainier, D. Y. Fibronectin regulates epithelial organization during myocardial migration in zebrafish. Dev. Cell 6, 371-382 (2004).

59. Qiao, L. et al. Snail modulates the assembly of fibronectin via $\alpha 5$ integrin for myocardial migration in zebrafish embryos. Sci. Rep. 4, 4470 (2014).

60. Jamora, C. et al. Regulation of Golgi structure through heterotrimeric G proteins. Cell 91, 617-626 (1997).

61. Wierzbicka-Patynowski, I., Mao, Y. \& Schwarzbauer, J. E. Analysis of Fibronectin Matrix Assembly. Current Protocols in Cell Biology (John Wiley and Sons, Inc., 2004).

62. Workman, P. et al. Guidelines for the welfare and use of animals in cancer research. Br. J. Cancer 102, 1555-1577 (2010).

\section{Acknowledgements}

We acknowledge Kenneth Yamada, Julia von Blume and Johanna Ivaska for providing reagents. We are grateful to Letizia Lanzetti and Julia von Blume for suggestions and insightful discussions. This work was supported by grants from: Telethon Italy (GGP09175) (to G.S.); Italian Association for Cancer Research (AIRC-IG Grants \#13016 and 16702 to G.S.); FPRC-ONLUS Grant 'MIUR 2010 Vaschetto-5 per mille 2010 MIUR' (to G.S.); Associazione 'Augusto per la Vita' (to G.S.); Ministero della Salute (RF-2011-02348194 to M.M.S.); ERC-CoN project 647057-rEnDOx (to M.M.S.); Deutsches Zentrum für Herz-Kreislauf-Forschung, partner site Munich Heart Alliance (to R.T.B.), the Deutsche Forschungsgemeinschaft (SFB914, Project A05 to R.T.B.).

\section{Author contributions}

D.V. and G.S. conceived the project; D.V., G.S., M.M.S., M.J.H., R.T.B., H.-Y.T. and M.R.M. designed the experiments; D.V., G.M., F.C., E.P., V.P., R.T.B., H.-Y.T., F.S., K.I.W. and M.R.M. performed the experiments; G.M., F.C., E.P., V.P., R.T.B., H.-Y.T., F.S., E.A., K.I.W., M.R.M., M.J.H., M.M.S., G.S. and D.V. analyzed the data; G.M., F.C. E.P., V.P., R.T.B., H.-Y.T., F.S., E.A., K.I.W., M.R.M., M.J.H., M.M.S., G.S. and D.V. interpreted the results; D.V., G.S., G.M., F.C., E.P. M.J.H. and M.M.S. wrote the paper; all authors read and approved the manuscript.

\section{Additional information}

Supplementary Information accompanies this paper at http://www.nature.com/ naturecommunications

Competing financial interests: The authors declare no competing financial interests.

Reprints and permission information is available online at http://npg.nature.com/ reprintsandpermissions/

How to cite this article: Mana, G. et al. PPFIA1 drives active $\alpha 5 \beta 1$ integrin recycling and controls fibronectin fibrillogenesis and vascular morphogenesis. Nat. Commun. 7, 13546 doi: $10.1038 /$ ncomms13546 (2016)

Publisher's note: Springer Nature remains neutral with regard to jurisdictional claims in published maps and institutional affiliations.

(c) (i) This work is licensed under a Creative Commons Attribution 4.0 International License. The images or other third party material in this article are included in the article's Creative Commons license, unless indicated otherwise in the credit line; if the material is not included under the Creative Commons license, users will need to obtain permission from the license holder to reproduce the material. To view a copy of this license, visit http://creativecommons.org/licenses/by/4.0/

(C) The Author(s) 2016 